\title{
Effects of toroidal plasma current on divertor power depositions on Wendelstein 7-X
}

Yu Gao ${ }^{1}$, Joachim Geiger ${ }^{2}$, Marcin W. Jakubowski²,3, Peter Drewelow ${ }^{2}$, Michael Endler $^{2}$, Kian Rahbarnia ${ }^{2}$, Sergey Bozhenkov ${ }^{2}$, Matthias Otte ${ }^{2}$, Yasuhiro Suzuki ${ }^{4,5}$, Yuhe Feng ${ }^{2}$, Holger Niemann ${ }^{2}$, Fabio Pisano ${ }^{6}$, Adnan Ali $^{2}$, Aleix Puig Sitjes ${ }^{2}$, Marco Zanini $^{2}$, Heinrich Laqua ${ }^{2}$, Torsten Stange ${ }^{2}$, Stefan Marsen ${ }^{2}$, Tamas Szepesi ${ }^{7}$, Daihong Zhang $^{2}$, Carsten Killer ${ }^{2}$, Kenneth Hammond ${ }^{2}$, Samuel Lazerson ${ }^{8}$, Barbara Cannas ${ }^{6}$, Henning Thomsen ${ }^{2}$, Tamara Andreeva ${ }^{2}$, Ulrich Neuner ${ }^{2}$, Jonathan Schilling ${ }^{2}$, Alexander Knieps ${ }^{1}$, Michael Rack ${ }^{1}$, Yunfeng Liang ${ }^{1}$, and the W7-X Team ${ }^{2}$

${ }^{1}$ Forschungszentrum Jülich GmbH, Institut für Energie- und Klimaforschung Plasmaphysik, Partner of the Trilateral Euregio Cluster (TEC), 52425 Jülich, Germany

${ }^{2}$ Max-Planck-Institut für Plasmaphysik, 17491 Greifswald, Germany

${ }^{3}$ University of Szczecin, Institute of Physics, 70-453 Szczecin, Poland

${ }^{4}$ National Institute for Fusion Science, National Institutes of Natural Sciences, Toki, Japan

${ }^{5}$ SOKENDAI (The Graduate University for Advanced Studies), Toki, Japan

${ }^{6}$ University of Cagliari, Department of Electrical and Electronic Engineering, 09123 Cagliari, Italy

${ }^{7}$ Wigner Research Center for Physics, Association EURATOM-HAS, H-1121, Budapest, Hungary

${ }^{8}$ Princeton Plama Physics Laboratory, NJ 08543 Princeton, U.S.A.

July 25, 2019

E-mail: y.gao@fz-juelich.de

\begin{abstract}
The paper presents experimental observations and simulations for the effects of toroidal plasma current on divertor power depositions on W7-X. With increasing toroidal current accompanying changes in the island geometry result in a sweep of the strike line and a redistribution of the heat flux footprints. Good agreement between experiments, which partly used electron cyclotron current drive to generate an additional toroidal current contribution, and modelling using field line tracing in vacuum magnetic fields including an ad-hoc toroidal current on the magnetic axis is found for both standard and low-iota magnetic configurations.
\end{abstract}

Keywords: toroidal current; heat flux; strike line; island divertor; W7-X

\section{Introduction}

The main goal of the stellarator Wendelstein $7-\mathrm{X}(\mathrm{W} 7-\mathrm{X})$ is to show that stellarators can be a viable concept for a steady-state operating fusion reactor. For this reason, the 
magnetic field configuration was developed in an optimization process to mitigate the main deficiencies of classical stellarator configurations. The resulting HELIAS-concept improves the equilibrium and stability properties, reduces neoclassical transport in the long-mean-free-path regime as well as the bootstrap current and enhances fast particle confinement at high plasma- $\beta$. The resulting magnetic configuration has five field periods with an aspect ratio of about 11 (major radius $R=5.5 \mathrm{~m}$, minor radius $a=0.5 \mathrm{~m}$ ). The standard magnetic configuration has a rotational transform $t$ just below 1 with a low positive shear in order to avoid neighboring major natural resonances in the profile. The coil system consists of 50 non-planar coils ( 5 coil types per half-period due to periodicity and stellarator-symmetry) and 20 planar coils ( 2 coil types per half-period) for additional flexibility as needed to change $t$ at the boundary of the vacuum configurations. For example, the so-called standard configuration has a boundary- $t$ value of 1 , which is accompanied by the $5 / 5$-island chain forming the plasma limiting separatrix, and the non-planar coils carry all the same current while the planar coils have none. With appropriate currents in the planar coils an additional toroidal field can be generated to lower the $t$-value so that the 5/6-resonance appears at the configuration boundary with the corresponding 5/6-island chain, the so-called low-iota configuration. Figure 1 shows magnetic geometries of the boundary islands in standard and low iota magnetic configurations. It should be noted that due to the periodicity the $5 / 5$ island chain consists of 5 separate islands while the 5/6-island chain is a single island winding around helically.

Apart from the necessary improvements of stellarator core plasma properties, a fusion reactor needs a particle and energy exhaust concept. For W7-X with its low-shear t-profile, the island divertor concept, which was tested successfully in W7-AS for the first time, has been chosen. It uses the naturally occurring 5/5-island chain as interaction topology with the divertor targets. For quasi-steady-state operation (1800 s pulses are envisioned) a fully cooled High-Heat-Flux (HHF-) divertor capable of withstanding $10 \mathrm{MW} \mathrm{m}^{-2}$ has been designed and is currently installed to be used from $2021 \mathrm{on}$. In two prior operational phases in 2017/18, OP1.2a and OP1.2b (OP abbreviating Operational Phase), an uncooled Test-Divertor-Unit (TDU) with the same geometry has been used in order to gain experience in machine operation and to explore plasma scenarios without the risk connected with water-cooled components in the vacuum vessel.

Although the target magnetic configuration in the optimization process of $\mathrm{W} 7-\mathrm{X}$ aimed at small bootstrap currents, this is generally not fully realized in all magnetic configurations and plasma scenarios. Due to the small shear in the $t$-profile, even rather small toroidal currents of a few $\mathrm{kAs}$ to a few tens of $\mathrm{kAs}$ lead to a considerable radial displacement of the boundary islands and thus change the interaction patterns of the magnetic field topology with the divertor components, i.e. the strike line patterns. Therefore, strike-line control, being one of the most important and challenging tasks for steady-state operation in any fusion device, shows to be an equally crucial task in a device with complex 3D magnetic topology at the plasma boundary like in Wendelstein $7-\mathrm{X}$ (W7-X) $[1,2,3]$.

In principle, a toroidal plasma current $I_{\text {tor }}$ is not favourable for low-shear stellarators, where the entire confining vacuum magnetic field is generated by external coils only, and where the low-shear $t$-profile is sensitive to small plasma currents. Thus, additional internal plasma currents can alter the anticipated magnetic structure and may lead to unfavourable modifications. $I_{\text {tor }}$ can arise as consequence of the intrinsic bootstrap current $I_{\text {bsc }}$, but also by any additional externally driven current $I_{\text {ext }}$ (e.g. electron cyclotron current drive (ECCD) [4]) and the transiently appearing plasma shielding currents $I_{\text {sld }}$. W7-X has no transformer to drive an ohmic current. The amplitude and radial profile of 
$I_{\text {bsc }}$ depend largely on the experimental parameters, e.g. magnetic configurations, heating power and plasma density. $I_{\mathrm{bsc}}$ is a toroidal net current and is predicted in W7-X to reach values of up to tens of $\mathrm{kA}$ for high-performance plasma discharges [5, 6] depending on the specific experimental scenario. The dependence of the bootstrap current on the magnetic configuration has been identified in a magnetic configuration scan discussed in 57]. The evolution of the toroidal current and the effect on the heat flux profile for the mimic configurations are shown in [8].

We are mainly concerned with the last one which determines the changes of the magnetic field at the plasma boundary, i.e. the radial position of the boundary islands for divertor interaction. This results in a ramp-up of the net $I_{\text {tor }}$ until a saturation at the amplitude of $I_{\mathrm{bsc}}+I_{\mathrm{ext}}$ is reached:

$$
I_{\mathrm{tor}}=I_{\mathrm{bsc}}+I_{\mathrm{ext}}+I_{\mathrm{sld}}=\left(I_{\mathrm{bsc}}+I_{\mathrm{ext}}\right)(1-\exp (-t R / L))
$$

In W7-X, a positive $I_{\text {tor }}$ will increase $t_{a}$ and move the boundary island chains towards the magnetic axis due to the positive shear at the plasma boundary. With the evolution of $I_{\text {tor }}$ during plasma operation, a change of strike-line locations is predicted depending on the magnetic configuration. Such strike line movements have been seen in plasma discharges throughout the divertor operation campaign OP1.2.

The experimental assessment of these effects uses quite different diagnostic capabilities of W7-X. $I_{\text {tor }}$ is measured experimentally by the in-vessel Rogowski coil encircling the plasma volume in W7-X $[9,10]$ to an accuracy in the range of several Ampères. The assessment of the interactions between the three dimensional (3D) magnetic island chains and the divertor plates, which results in toroidally asymmetric but stellarator-symmetric power loads [11], requires a complete coverage of plasma facing component by real-time video diagnostics, which is also necessary for safe operation. Ten infrared (IR) thermographic systems with wide-angle optics were installed to monitor the surface temperature on the carbon divertor plates in all the ten half modules in the machine $[12,13,14,15]$. First characterizations of the thermal footprints on the target have been in good agreement with results from field line tracing (FLT) for different vacuum magnetic configurations $[16,17]$, which give confidence in using these tools in the current assessment. The methods to quantitatively analyze and visualize the heat loads on the TDUs measured by IR cameras as well as to compare with diffusive FLT-results have been introduced in [18] and are used throughout this paper. To investigate the correlation between the experimental divertor thermal footprints and the measured $I_{\text {tor }}$, simulation results are used which are obtained with a field line tracer [19] available as web-service at IPP.

In section 2 , a typical freely-evolving $I_{\mathrm{bsc}}$ program in the standard magnetic configuration of $\mathrm{W} 7-\mathrm{X}$ will be investigated and a quantitative analysis of the strike-line patterns on the divertor target plates is performed. Corresponding simulations will be shown and compared with experimental results in section 3. In section 4, the same comparisons are done in the low-iota configuration which confirm the effects of $I_{\text {tor }}$ seen in the previous section. Finally, in section 5, we will show results from dedicated ECCD experiments suggesting it to be one possible candidate for a dynamic control of $I_{\text {tor }}$ in the future. 


\section{Experimental observations in the standard mag- netic configuration}

The standard reference magnetic configuration was one of the most used magnetic configurations for divertor studies at $t_{a}=1$ with the $5 / 5$ boundary island chain limiting the plasma. Because of the sensitivity of this configuration to the 1/1-error fields the additional so-called trim coils were used to compensate the major part of this error field component $[20,21]$. However, a small part of the error field, a 2/2-component, remained. Thus, this configuration does not show perfect periodicity and the integral power deposition compared among machine modules is not completely symmetrized. But the general behavior of the strike-line dynamics with evolving $I_{\text {tor }}$ observed during experiments is quantitatively the same across modules.

To study the effect of $I_{\text {tor }}$ on the strike-line movement we used discharges performed for bootstrap current investigations. A typical bootstrap current program (20171108.040) was operated with helium plasma and ECRH heating power $P_{\mathrm{ECRH}}$ of $3.6 \mathrm{MW}$. An overview of the discharge parameters can be seen in figure 2 . The measured $I_{\text {tor }}$ ramps up to $\sim 6 \mathrm{kA}$ within $10 \mathrm{~s}$. The intermittent spikes in the $P_{\mathrm{ECRH}}$ are due to a gyrotron operated in blip mode for the measurement of collective Thomson scattering. The discharge has only a pre-fill of the helium gas and no further gas feed, which causes a continuous drop of the line integrated electron density $\int n_{\mathrm{e}} d l$ from $3.9 \times 10^{19}$ to $3 \times 10^{19} \mathrm{~m}^{-2}$ through pumping. During the operation of this discharge, both strike lines on the horizontal and vertical targets were observed to move away from the pumping gap. In order to quantitatively determine the relation between the strike-line motion and the increasing $I_{\text {tor }}$, a series of detailed analysis based on the thermographic method developed in [18] are performed here.

The visualized footprints of the heat fluxes on the upper divertor target plate in module $5(5 \mathrm{u})$ at $1 \mathrm{~s}\left(I_{\text {tor }}=1 \mathrm{kA}\right)$ and at $9 \mathrm{~s}\left(I_{\text {tor }}=5.5 \mathrm{kA}\right)$ are shown in figure 3 . Compared with the $1 \mathrm{kA}$ case, the strike lines of the $5.5 \mathrm{kA}$ are, on both the horizontal and vertical targets, shifted further away from the pumping gap. In the lower part of figure 3 we marked target fingers which had been installed for plasma-wall-interaction (PWI) studies. The calculated heat fluxes for these fingers are arbitrary due to the complex surface layers which have been prepared intentionally on the marker tiles for material studies. But the temperature measurements for these fingers are valid, which will be shown in section 3 . The indices of the fingers are counted starting from the low-iota end of the divertor (left in the figure) with the target finger 0 (e.g. 5uh_l_11 means module 5, upper horizontal target, low-iota part, finger 11). The low-iota part of the TDU is the region, where most power loads will be deposited in a low iota magnetic configuration (area shown in [18]. The hot spot marked in the figure is considered to be caused by particle drifts [22], because it is updown asymmetric and the asymmetry reverses in experiments with reversed field. This effect is out of the scope of this paper. The influence of the PWI fingers and of the hot spot on the heat flux distributions is excluded from the analysis in this paper since we are aiming to understand only the $I_{\text {tor }}$ effect.

The redistribution of the $3 \mathrm{D}$ heat fluxes by $I_{\text {tor }}$ can be seen in figure 3 . With $I_{\text {tor }}$ increasing from $1 \mathrm{kA}$ to $5.5 \mathrm{kA}$, more power is deposited onto the vertical target, while less power reaches the horizontal target. To more clearly show the movements of the heat fluxes of the main strike lines (avoiding the PWI elements and hot spot), two fingers are used for a detailed analysis (5uh_l_11 for the upper, horizontal, low-iota part of the divertor and 5uv_06 for the upper, vertical target as marked in figure 3). The peak heat flux $q_{\mathrm{h}}$ on 
the finger of the horizontal target 5uh_l_11 decreases from $1.4 \mathrm{MW} \mathrm{m}^{-2}$ to $0.75 \mathrm{MW} \mathrm{m}^{-2}$, while the peak heat flux $q_{\mathrm{v}}$ of the vertical target finger 5uv_06 increases from $1.1 \mathrm{MW} \mathrm{m}^{-2}$ to $1.6 \mathrm{MW} \mathrm{m}^{-2}$. This results in an increased ratio of $q_{\mathrm{v}}$ to $q_{\mathrm{h}}$ by a factor of 2.7. This redistribution of heat fluxes is due to the geometric effect of the movement of the divertor intersecting island by the increasing $I_{\text {tor }}$, which can be reproduced by simulations (see section 3$)$.

The movement of the strike lines is rather homogeneous toroidally over time, as shown in figure 4. For each finger, multiple profiles along the poloidal length of the finger are interpolated from experimental data for heat flux calculations [18]. Here, the peaks of these heat flux profiles on the low-iota part of the horizontal target are plotted for different time points in the discharge. The corresponding toroidal angle for each radial profile is calculated for a better comparison with simulation result. The location in the figure is defined as the distance from the edge of each finger at the pumping gap. Based on this observation, it seems to be feasible to represent the strike-line motions as a whole using one specific finger only. In order to correlate quantitatively the strike-line movement with the changes in $I_{\text {tor }}$ with a better statistic, 15 lines of the heat flux profiles within finger 5uh_l_11 ( with the central line of the finger at toroidal angle $\phi=-60^{\circ}$ ) are averaged. In figure 5 the time evolution of the heat flux shows a continuous movement of the strike line (maximum position of heat flux) outward with increasing $I_{\text {tor }}$, as well as a decrease of the amplitude of the heat flux.

To locate the peak of the heat flux of the strike line accurately, a polynomial least square fitting procedure has been performed to all averaged heat flux profiles of the finger between $1 \mathrm{~s}$ and $9 \mathrm{~s}$ of this discharge. Figure 6 shows an example of ten profiles with peak locations derived from the polynomial fits marked with crosses. As already described, we see that the strike line moves outward with time, the peak heat flux reduces and the wetted area broadens. A plot of the peak locations against $I_{\text {tor }}$ is presented in figure 7 , where the data points are collected from 875 frames captured by the IR camera in this discharge. The strike line moves almost linearly with $I_{\text {tor }}$ with an average ratio of distance to $I_{\text {tor }}$ of $\sim 9 \mathrm{~mm} \mathrm{kA}^{-1}$.

\section{Simulations compared with experiments in stan- dard magnetic configuration}

The simulations performed in this paper use field line tracing (FLT) in the vacuum magnetic field provided at IPP as a web-service and approximate the additional $I_{\text {tor }}$ with a current filament along the magnetic axis. This approximation serves well for the comparison with experiments at low plasma- $\beta$ and with limited values of $I_{\text {tor }}(<10 \mathrm{kA}$ at $B_{0}=2.5 \mathrm{~T}$ and volume-averaged $\beta$-values $\leq 1 \%$ in OP1.2). Preliminary transport analysis using the NTSS-code [23] also shows that the bootstrap current as well as the shielding currents are, due to the peaked electron temperature profiles, more centrally distributed, thus justifying our approach. However, for simulations with higher $I_{\text {tor }}$ or higher $\beta$-values, comprehensive equilibrium fields $[24,25,26]$ need to be used as they are provided by the VMEC/EXTENDER-approach [5, 6] or by 3D-equilibrium codes like HINT [27, 28], PIES [25], SIESTA [29] or SPEC [30].

Figure 8 shows simulations of the wall to wall connection length $\left(L_{c}\right)$ of magnetic field lines in the poloidal cross section at $\phi=-60^{\circ}$ with different $I_{\text {tor }}$. The increasing $I_{\text {tor }}$ moves the boundary island chain towards the magnetic axis, and as a result, shifts the 
strike lines away from the pumping gap. With the movement of the magnetic island, the region of the island around the island-O-point with $L_{c}>1 \mathrm{~km}$ becomes larger, since more flux surfaces of the island are no longer intersected by divertor components as $I_{\text {tor }}$ increases.

For a quantitative comparison with the experimental results, the $L_{c}$ profiles for the same finger having been analyzed in section $2\left(5 \mathrm{uh} \_l \_11\right)$ are averaged for different $I_{\text {tor }}{ }^{-}$ values and this $L_{c}$-evolution is shown in figure 9. The peak locations of $L_{c}$ are used as representative for the simulated strike-line locations although its profiles differ considerably from the experimentally measured heat flux profiles due to heat diffusive transport. The strike line moves away from the pumping gap with increasing $I_{\text {tor }}$ reflecting the experimental results. Additionally, the value of $L_{c}$ increases from $\sim 580 \mathrm{~m}$ to $\sim 780 \mathrm{~m}$ with an increase of $I_{\text {tor }}$ from $0 \mathrm{kA}$ to $6 \mathrm{kA}$. The larger values of $L_{c}$ are consistent with the broader strike-line width or wetted area on the divertor plate [16]. This is the main reason for the broadening of the experimental heat flux profiles over time seen in figure 6 . The simulated and the experimental strike-line locations are compared in figure 7 . The reasonable agreement between the results from experiment and from simulation strengthens the interpretation of the strike-line motion via the $I_{\text {tor-effect. }}$

More sophisticated simulations are required to better understand the redistribution of thermal loads by changes of $I_{\text {tor }}$. In a first but simple approach we use FLT with a diffusion ansatz [19] to simulate plasma transport effects. For this purpose test particles are placed initially inside the last closed flux surface but in its vicinity. They are generated by five FLT-runs to preserve the five fold periodicity of the W7-X magnetic field starting from different periods of the machine to ensure the toroidally symmetric distribution. The diffusion process is performed with a perpendicular diffusion coefficient $D_{\perp}=1 \mathrm{~m}^{2} \mathrm{~s}^{-1}$, a mean free path parameter $\lambda=0.1 \mathrm{~m}$, and a velocity $v=2 \times 10^{6} \mathrm{~m} \mathrm{~s}^{-1}$ which corresponds to a magnetic diffusion coefficient $D_{m}=2 \times 10^{-6} \mathrm{~m}^{2} \mathrm{~s}$ as defined in [31]. A scan of these input parameters has been performed earlier aiming for a best fit between simulated and experimental strike-line widths as shown in figure 10 and as had been suggested in [31]. The test particles are traced until hitting a component where they are recorded as seen in figure 11 which compares the results of this "diffusive" FLT of the 1 and the $5.5 \mathrm{kA}$ cases. The simulations show the strike lines to be further away from the pumping gap with larger $I_{\text {tor }}$, which is consistent with the experimental observations.

Due to the symmetry of the divertor (5-fold periodicity and stellarator-symmetry) the resulting test particle distributions of the ten half modules can be merged into one improving the statistics. For the calculation of the heat fluxes on the TDU, we assume a total power of $1.2 \mathrm{MW}$ is equally distributed to the $\sim 75000$ test particles, which have been traced to intersect with the TDU. This assumed power shared by the test particles has been determined by fitting the simulated heat flux amplitudes to the experimental ones. With the same method as described in [18], the simulated distribution of the heat fluxes on the target plates are visualized as shown in figure 12. Compared with the experimental results in figure 3, the simulations qualitatively reproduce the thermal footprints in terms of the strike-line movements and the increased ratio of $q_{\mathrm{v}}$ to $q_{\mathrm{h}}$, caused by the increasing $I_{\text {tor }}$. However, the model of "diffusive FLTs" does not account for particle drifts nor for local plasma radiation, so that a perfect match with the experimental heat flux distributions is not to be expected.

An interesting feature in the simulations is the appearance of the second strike line on the horizontal target, which is also observed in the experiment as shown in the $5.5 \mathrm{kA}$ case in figure 3. By splitting up the test particles intersecting the TDU based on the direction 
of the tracing, forward being the same direction as the toroidal field and backward being the counter-direction, one can distinguish the corresponding footprints of co- and counterflow test particles as shown at the bottom of figure 12 for $5.5 \mathrm{kA}$ case. The simulation suggests that the second strike line is caused by plasma flowing in the opposite toroidal direction compared to the plasma causing the main strike line. With respect to the heat flux on the vertical target, the counter flowing plasma also contributes to the additional heat load. To better understand this phenomena, figure 13 shows a plot of $L_{c}$ for the case with $I_{\text {tor }}=5.5 \mathrm{kA}$ at a toroidal angle of $\phi=-68^{\circ}$. The toroidal angle has been chosen according to the divertor finger (5uh_l_25) which is marked in figure 3, where a footprint of the second strike line is expected to appear. The poloidal cross section shows that the upper island is only barely intersected by the horizontal target, i.e. the island chain is almost lifted off from the divertor plate with the increasing $I_{\text {tor }}$. Compared with $\phi=-60^{\circ}$ (figure 8 with $5500 \mathrm{~A}$ ), at $\phi=-68^{\circ}$ the main strike line of the upper island is even closer to the pumping gap, and no intersection exists between the vertical target and the inboard island. These toroidal variations are due to the complex interplay between 3D magnetic configuration and divertor geometry in W7-X, and are consistent with experimental observations in figure 3, where toroidal asymmetric power distributions on the targets are demonstrated. Moreover cross field transport can deposit heat loads on the horizontal target from the outboard leg of the island with $L_{c} \sim 500 \mathrm{~m}$ forming the second strike line. Plasma flowing on this leg is no longer intersected by other in-vessel components as the island chains move towards the magnetic axis. This second strike line generated by the counter flow could be confirmed directly in experimental observations. Figure 14 shows the distribution of the temperature rise on the surface of a PWI finger (5uh_l_19) from the beginning of the discharge to $9 \mathrm{~s}$ ( $5.5 \mathrm{kA}$ case). A small misalignment during the installation of this finger makes this finger stick out slightly between the neighboring ones generating leading edges on both sides [32]. Thus, this finger is capable to visualize flow coming from both directions. The opposite directions of the temperature gradients seen for the main strike line and the second one on this finger confirms the simulation results.

While the second strike line is clearly visible in the plot of the test particles hitting the divertor targets (lowest sub-figure in figure 12), the heat flux calculated from the simulation (middle sub-figure in figure 12) shows the second stripe less prominently when compared with the experimental results. Figure 10 shows a quantitative comparison for the two different fingers (5uh_l_11 and 5uh_l_25) with $I_{\text {tor }}$ of 1 and $5.5 \mathrm{kA}$. The locations of the maxima and the peak amplitudes agree rather well, however, differences can also be observed, especially for the second strike line in the $5.5 \mathrm{kA}$-case which is barely identifiable in the simulations. Also for finger $5 \mathrm{uh} \_\mathrm{l} 11$ at $5.5 \mathrm{kA}$, the experimental result shows a larger decay length than the simulation. This is due to the merging of the second strike line with the main strike line at $\phi=-60^{\circ}$ which is also seen in the experimental map of the heat flux in figure 3. Although the second strike line in the plot of the test particle simulation (blue dots in fig. 11) extends to $\phi=-60^{\circ}$, there are so few points hitting the divertor targets that they only contribute negligibly to the amplitude of the simulated heat flux at 5uh_l_11 compared with the experimental results. Also for the finger 5uh_l_25 at $5.5 \mathrm{kA}$ at the local position of $0.25 \mathrm{~m}$ on the finger, the experimental heat flux profile shows a secondary peak with a heat flux of $0.23 \mathrm{MW} \mathrm{m}^{-2}$, while in the simulation there are only $0.1 \mathrm{MW} \mathrm{m}^{-2}$ seen. The main reason for these differences is considered to be the particle drifts, i.e. $\mathbf{E} \times \mathbf{B}$ and/or $\mathbf{B} \times \nabla B$, which are not included in the simulation. The simulation presented here uses only an isotropic diffusion coefficient $D_{\perp}$, while in reality 
particle drifts add convection patterns to the edge transport, such that the second leg of the island can get more loads. An upgraded version of the comprehensive edge simulation code EMC3-EIRENE [33, 34] is foreseen to incorporate drifts to quantitatively understand this effect in the future [35].

\section{Experimental results and simulations in the low- iota configuration}

The boundary topology of the low-iota configuration is formed by the island of the $5 / 6$ resonance in the $t$ profile. In contrast to the five separate islands of the standard configuration, the 5/6-island is one flux tube, i.e. all six islands appearing in the Poincaré plot of a poloidal cross section are connected helically. Also the radial width of the $5 / 6$-islands is smaller than the one of the $5 / 5$-islands and due to the higher poloidal mode number, the poloidal extent of the island and therefore the strike-line distribution is different which is also connected to the different $t_{a}$-value. Calculations of $L_{c}$ for 1 and for $3 \mathrm{kA}$ at the cross section at $\phi=80^{\circ}$ are compared in figure 15 and exemplify the radial displacement of the island and the corresponding movement of the strike line. Compared with the standard configuration, the outboard-side leg of the island is intersecting the target plate. With the island moving towards the magnetic axis with increasing $I_{\text {tor }}$, the strike line is shifting towards the pumping gap on the horizontal target.

We apply the same strike line analysis of the previous sections to both experimental and simulation data for a typical low-iota configuration discharge (XP:20180829_009) with $P_{\mathrm{ECRH}}=5 \mathrm{MW}$ and $\int n_{\mathrm{e}} d l=2.4 \times 10^{19} \mathrm{~m}^{-2}$. The thermal footprint of the strike line on the divertor is shown in figure 16 for overview purposes, and we marked the finger 2uh_l_16 at $\phi=80^{\circ}$ used for the detailed analysis. In this discharge $I_{\text {tor }}$ ramps up to $3 \mathrm{kA}$, which leads to a slight inward shift of the strike line which is seen in figure 17, where we show the time evolution of the averaged heat flux of the chosen divertor target finger as well as the time traces of $I_{\text {tor }}$ and of the ECRH-power. Since the boundary topology of this configuration is not sensitive to error fields, we can compare the experimental results of all available IR cameras for this discharge more confidently. Figure 18 shows the strike-line locations at the identical positions of the fingers (XXh_l_16) in each half module plotted against $I_{\text {tor }}$. Due to particle drift effects, the locations of the experimental strike lines on all the upper targets are displaced further out by $\sim 3 \mathrm{~cm}$ compared to the lower targets. The maxima of the simulated heat fluxes are located between the experimental results. There are two deviations in the movement of the strike-line of divertor module 5uh which is due to the perturbation in the downstream heat fluxes by the deep plunges of the multi-purpose manipulator probe [36] at an upstream position. Overall, a quantitative agreement between the simulated and experimental strike-line movements against $I_{\text {tor }}$ for the low-iota configuration is found.

\section{$5 \quad$ ECCD effects on $I_{\text {tor }}$}

Dedicated ECCD programs had been performed to modify the $I_{\text {tor }}$. Figure 19 shows the time evolution of the averaged heat flux on finger 5uh_l_11 for a discharge without ECCD (20170927_028 (top)), a counter-ECCD discharge 030 (middle) and co-ECCD discharge 042 (bottom) in standard magnetic configuration. To clarify the notation, co-ECCD increases $t_{a}$, while counter-ECCD decreases $t_{a}$. In the no-ECCD case $I_{\text {tor }}$ ramps up to 
$1 \mathrm{kA}$, while for co-ECCD $4 \mathrm{kA}$ are reached finally and in the counter-ECCD case $I_{\text {tor }}$ decreases to a slightly negative value. In the co-ECCD example, the toroidal current driven in the core is changing the $t$ profile in the confined region, which leads to magnetohydro-dynamic (MHD) instabilities [2] affecting the confinement and generally clearly seen in the electron temperature measurements by ECE. The Co-ECCD-case in figure 19 shows three of these fast events which deposit also a large amount of heat flux after $1.25 \mathrm{~s}$ on the divertor and which are correlated with fast crashes of the electron temperature in the core. The strike-line movement with $I_{\text {tor }}$ in these scenarios is plotted in figure 20. It is clear that despite the fast events (marked in the figure), the strike line movement correlates well with $I_{\text {tor }}$ almost linearly with an averaged displacement to $I_{\text {tor }}$ ratio of $\sim 10 \mathrm{~mm} \mathrm{kA}^{-1}$. A discussion of these MHD instabilities is out of the scope of this paper. We note that the ECCD discharges used different trim coil [37] settings for error field correction compared with program 20171108_040 shown in section 2 with the freely evolving bootstrap current. Therefore, a direct comparison of the strike-line locations between them is not possible.

\section{Conclusion and discussion}

The effects of a toroidal plasma current on the power deposition on the divertor of W7$\mathrm{X}$ has been studied in this paper. First, simulations and experimental measurements are compared in a typical discharge in the standard magnetic configuration with $I_{\text {tor }}$ ramping up to $6 \mathrm{kA}$. The strike-line movement has been found to be consistent with field line diffusion simulations using the vacuum field and approximating the effect of $I_{\text {tor }}$ by adding the toroidal current as a filament current on the magnetic axis. At the toroidal angle of $\phi=-60^{\circ}$, the strike line has been studied in more detail and has been observed to move almost linearly with the ratio of the average movement to $I_{\text {tor }}$ by about $\sim 9 \mathrm{~mm} / \mathrm{kA}$. Also, with increasing $I_{\text {tor }}$, the vertical target is observed to receive more heat load than the horizontal target. All in all, the simulated divertor heat fluxes show qualitative agreement with the experimental results. We also observe the appearance of a second strike line, which is also seen in simulations. The simulations show that this is due to the heat transport from the outboard leg of the island, which is no longer shielded by other invessel components as the island chains moves towards the magnetic axis with increasing $I_{\text {tor }}$. The observed reduction of the peak heat flux as well as the broadening of the heat flux profile on the horizontal target is mainly caused by the modified geometry of the island with increasing $I_{\text {tor }}$. On the other hand, in the low-iota magnetic configuration, an inward shift of the strike lines with increasing $I_{\text {tor }}$ is observed. This is consistent with simulations which show that the visible stike line is the outboard leg of the target intersecting island and the island displacement towards the magnetic axis moves the strike line to the inboard side. Experiments dedicated to electron cyclotron current drive (ECCD) have also been used for investigations of the strike line movements. $I_{\text {tor }}$ being modified by ECCD shows the same strike line changes as the experiments with the freely evolving current. This suggests that ECCD may be a possible candidate for the strike-line control in the future.

We note, that there are several limitations of the simulation that have to be discussed. First, the method applied in the paper to use a helical current filament on the axis together with the vacuum fields is not applicable for larger values of $I_{\text {tor }}>10 \mathrm{kA}$. This is due to the modifications of $t$ profile which becomes particularly important for larger toroidal currents when island chains can appear further out in the confinement volume due to low order rational values. For such cases, equilibrium calculation codes such as VMEC [38] which excludes the existence of islands in the equilibrium region, or more ad- 
vanced codes allowing for their existence like PIES [39, 25], HINT [40, 27, 28], SIESTA [29] or SPEC [30] have to be used for the simulation with higher $I_{\text {tor }}$. In such cases complete limiter configuration can develop, since the further increase of $t_{a}$ will move the islands so far inwards into the plasma that they intersect with the divertor and good flux surfaces can enclose them. Second, particle drifts have not been included in the simulations and they are considered to be the main reason for the differences between the simulated and experimental divertor thermal footprints on the divertor plates (e.g. the hot spot and the less populated second strike line). Moreover, while the low-iota magnetic configuration is not sensitive to error fields like the $n / m=1 / 1$, the residual error fields have to be understood before simulations in the standard magnetic configuration with $t=1$ at the boundary can be compared with experiments on all the divertor targets. Finally, a more complete treatment of the SOL-transport will be needed to understand the details of the experiments. This will need simulations with SOL-transport codes like EMC3-EIRENE which also consider impurity and neutral transport, which is foreseen for the future.

\section{Acknowledgments}

This work has been carried out within the framework of the EUROfusion Consortium and has received funding from the Euratom research and training programme 2014-2018 and 2019-2020 under grant agreement No 633053. The views and opinions expressed herein do not necessarily reflect those of the European Commission.

\section{References}

[1] T. Klinger, A. Alonso, S. Bozhenkov et al. Performance and properties of the first plasmas of Wendelstein 7-X. Plasma Physics and Controlled Fusion, 59 (1), 014018 (2017).

[2] R. Wolf, A. Ali, A. Alonso et al. Major results from the first plasma campaign of the Wendelstein 7-X stellarator. Nuclear Fusion, 57 (10), 102020 (2017).

[3] T. Sunn Pedersen, A. Dinklage, Y. Turkin et al. Key results from the first plasma operation phase and outlook for future performance in Wendelstein 7-X. Physics of Plasmas, 24 (5), 055503 (2017).

[4] T. Stange, H. Laqua, M. Beurskens et al. Advanced electron cyclotron heating and current drive experiments on the stellarator Wendelstein 7-X. EPJ Web Conf., 157, 02008 (2017).

[5] J. Geiger, C. Beidler, M. Drevlak et al. Effects of Net Currents on the Magnetic Configuration of W7-X. Contributions to Plasma Physics, 50 (8), 770 (2010).

[6] J. Geiger, C. Beidler, M. Drevlak et al. Erratum: Effects of Net Currents on the Magnetic Configuration of W7-X. Contributions to Plasma Physics, 51 (1), 99 (2011).

[7] A. Dinklage, C. Beidler, P. Helander et al. Magnetic configuration effects on the Wendelstein 7-X stellarator. Nature Physics, 14 (8), 855 (2018). 
[8] J. Lore, Y. Gao, J. Geiger et al. Measurement and modeling of magnetic configurations to mimic overload scenarios in the W7-X stellarator. Nuclear Fusion, 59 (6), 066041 (2019).

[9] M. Endler, B. Brucker, V. Bykov et al. Engineering design for the magnetic diagnostics of Wendelstein 7-X. Fusion Engineering and Design, 100, 468 (2015).

[10] K. Rahbarnia, H. Thomsen, U. Neuner et al. Diamagnetic energy measurement during the first operational phase at the Wendelstein 7-X stellarator. Nuclear Fusion, 58 (9), 096010 (2018).

[11] Y. Feng, F. Sardei, P. Grigull et al. Physics of island divertors as highlighted by the example of W7-AS. Nuclear Fusion, 46 (8), 807 (2006).

[12] M. Jakubowski, P. Drewelow, J. Fellinger et al. Infrared imaging systems for wall protection in the W7-X stellarator (invited). Review of Scientific Instruments, 89 (10), 10E116 (2018).

[13] A. Puig Sitjes, M. Jakubowski, A. Ali et al. Wendelstein 7-X Near Real-Time Image Diagnostic System for Plasma-Facing Components Protection. Fusion Science and Technology, 74 (1-2), 116 (2018).

[14] A. Ali, M. Jakubowski, H. Greuner et al. Experimental results of near real-time protection system for plasma facing components in Wendelstein 7-X at GLADIS. Physica Scripta, 2017 (T170), 014074 (2017).

[15] F. Pisano, B. Cannas, M. W. Jakubowski et al. Towards a new image processing system at Wendelstein 7-X: From spatial calibration to characterization of thermal events. Review of Scientific Instruments, 89 (12), 123503 (2018).

[16] M. Jakubowski, A. Ali, P. Drewelow et al. 3D heat and particle fluxes in Wendelstein 7-X. 27th IAEA Fusion Energy Conference (2018).

[17] T. S. Pedersen, R. König, M. Krychowiak et al. First results from divertor operation in Wendelstein 7-X. Plasma Physics and Controlled Fusion, 61 (1), 014035 (2019).

[18] Y. Gao, M. W. Jakubowski, P. Drewelow et al. Methods for quantitative study of divertor heat loads on W7-X. Nuclear Fusion, 59 (6), 066007 (2019).

[19] S. Bozhenkov, J. Geiger, M. Grahl et al. Service oriented architecture for scientific analysis at W7-X. An example of a field line tracer. Fusion Engineering and Design, 88 (11), 2997 (2013).

[20] S. Bozhenkov, M. Otte, C. Biedermann et al. Measurements and correction of the 1/1 error field in Wendelstein 7-X. Nuclear Fusion, 59 (2), 026004 (2018).

[21] S. A. Lazerson, S. Bozhenkov, B. Israeli et al. Error fields in the Wendelstein 7-X stellarator. Plasma Physics and Controlled Fusion, 60 (12), 124002 (2018).

[22] K. C. Hammond, P. Drewelow, P. Drews et al. The role of particle drifts in W7-X divertor heat load asymmetries. Plasma Physics and Controlled Fusion (submitted).

[23] Y. Turkin, C. D. Beidler, H. Maaberg et al. Neoclassical transport simulations for stellarators. Physics of Plasmas, 18 (2), 022505 (2011). 
[24] P. Helander, C. D. Beidler, T. M. Bird et al. Stellarator and tokamak plasmas: a comparison. Plasma Physics and Controlled Fusion, 54 (12), 124009 (2012).

[25] M. Drevlak, D. Monticello and A. Reiman. PIES free boundary stellarator equilibria with improved initial conditions. Nuclear Fusion, 45 (7), 731 (2005).

[26] Y. Suzuki and J. Geiger. Impact of nonlinear 3D equilibrium response on edge topology and divertor heat load in Wendelstein 7-X. Plasma Physics and Controlled Fusion, 58 (6), 064004 (2016).

[27] Y. Suzuki, N. Nakajima, K. Watanabe et al. Development and application of HINT2 to helical system plasmas. Nuclear Fusion, 46 (11), L19 (2006).

[28] Y. Suzuki. HINT modeling of three-dimensional tokamaks with resonant magnetic perturbation. Plasma Physics and Controlled Fusion, 59 (5), 054008 (2017).

[29] H. Peraza-Rodriguez, J. M. Reynolds-Barredo, R. Sanchez et al. Bootstrap current control studies in the Wendelstein 7-X stellarator using the free-plasma-boundary version of the SIESTA MHD equilibrium code. Plasma Physics and Controlled Fusion, 60 (2), 025023 (2018).

[30] S. R. Hudson, R. L. Dewar, G. Dennis et al. Computation of multi-region relaxed magnetohydrodynamic equilibria. Physics of Plasmas, 19 (11), 112502 (2012). Base reference SPEC together with PPCF-article which is a little bit earlier. This is the more fundamental and more elaborate article.

[31] J. D. Lore, M. Cianciosa, H. Frerichs et al. Modeling and Preparation for Experimental Testing of Heat Fluxes on W7-X Divertor Scraper Elements. IEEE Transactions on Plasma Science, 46 (5), 1387 (2018).

[32] M. Endler, J. Fellinger, H. Hölbe et al. Managing leading edges during assembly of the Wendelstein 7-X divertor. Plasma Physics and Controlled Fusion, 61 (2), 025004 (2018).

[33] Y. Feng, F. Sardei, J. Kisslinger et al. 3D Edge Modeling and Island Divertor Physics. Contributions to Plasma Physics, 44 (13), 57 (2004).

[34] D. Reiter, M. Baelmans and P. Brner. The EIRENE and B2-EIRENE Codes. Fusion Science and Technology, 47 (2), 172 (2005).

[35] F. Schluck, M. Rack, D. Reiter et al. On the effects of kinetic minority ions on transport in Wendelstein 7-X. In 45th EPS Conference on Plasma Physics (2018).

[36] D. Nicolai, V. Borsuk, P. Drews et al. A multi-purpose manipulator system for W7-X as user facility for plasma edge investigation. Fusion Engineering and Design, 123, 960 (2017). Proceedings of the 29th Symposium on Fusion Technology (SOFT-29) Prague, Czech Republic, September 5-9, 2016.

[37] T. Rummel, K. Risze, J. Kiszlinger et al. The Trim Coils for the Wendelstein 7-X Magnet System. IEEE Transactions on Applied Superconductivity, 22 (3), 4201704 (2012). 
[38] S. Hirshman, W. van RIJ and P. Merkel. Three-dimensional free boundary calculations using a spectral Green's function method. Computer Physics Communications, 43 (1), 143 (1986).

[39] A. H. Reiman. Suppression of magnetic islands by rf driven currents. The Physics of Fluids, 26 (5), 1338 (1983).

[40] K. Harafuji, T. Hayashi and T. Sato. Computational study of three-dimensional magnetohydrodynamic equilibria in toroidal helical systems. Journal of Computational Physics, 81 (1), 169 (1989).

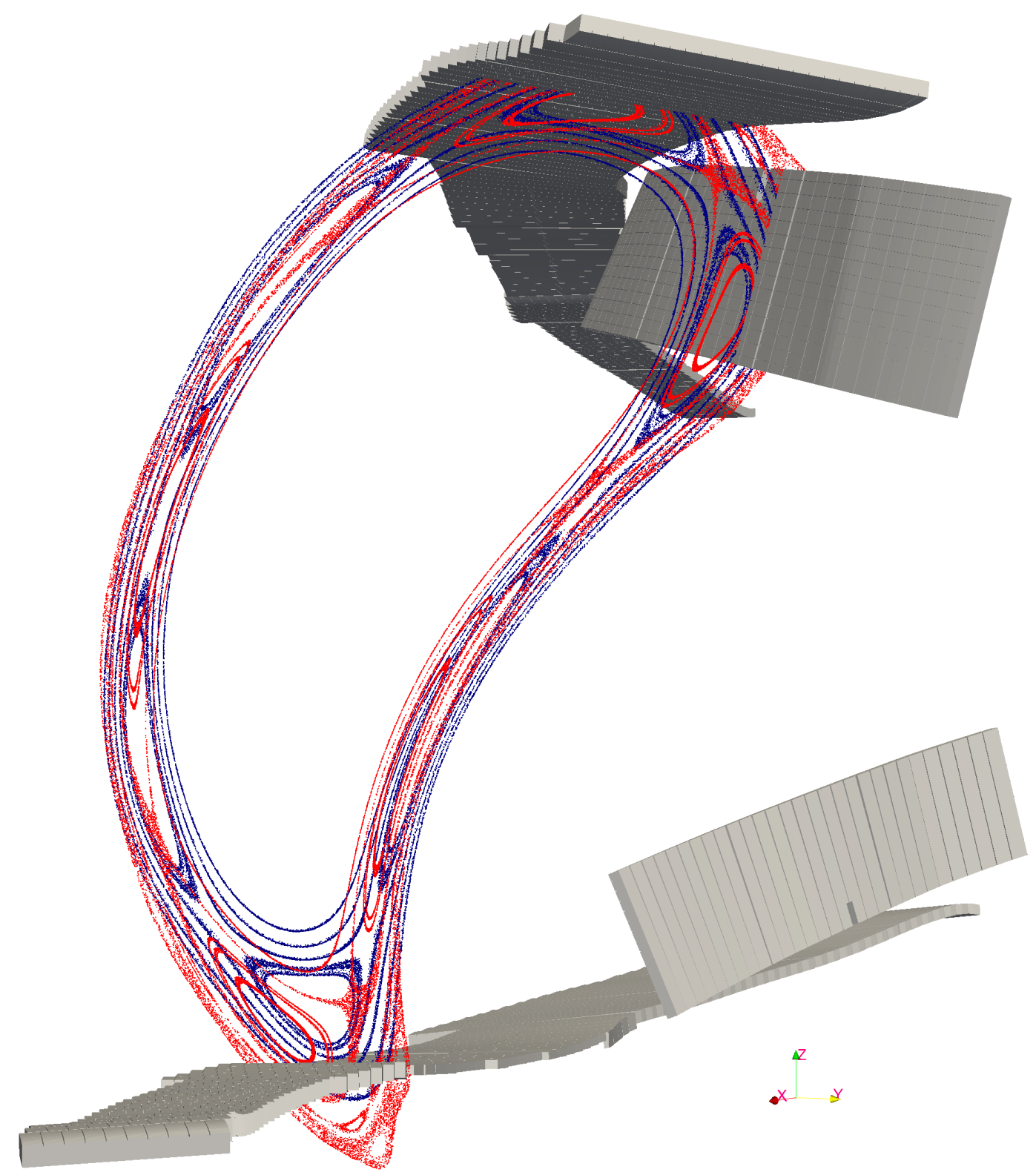

Figure 1: Poincaré plots at toroidal angle $\phi=-60^{\circ}$ for standard (red) and low iota (blue) magnetic configurations as well as the Test-Divertor-Units (TDUs) in one of the five machine modules. 


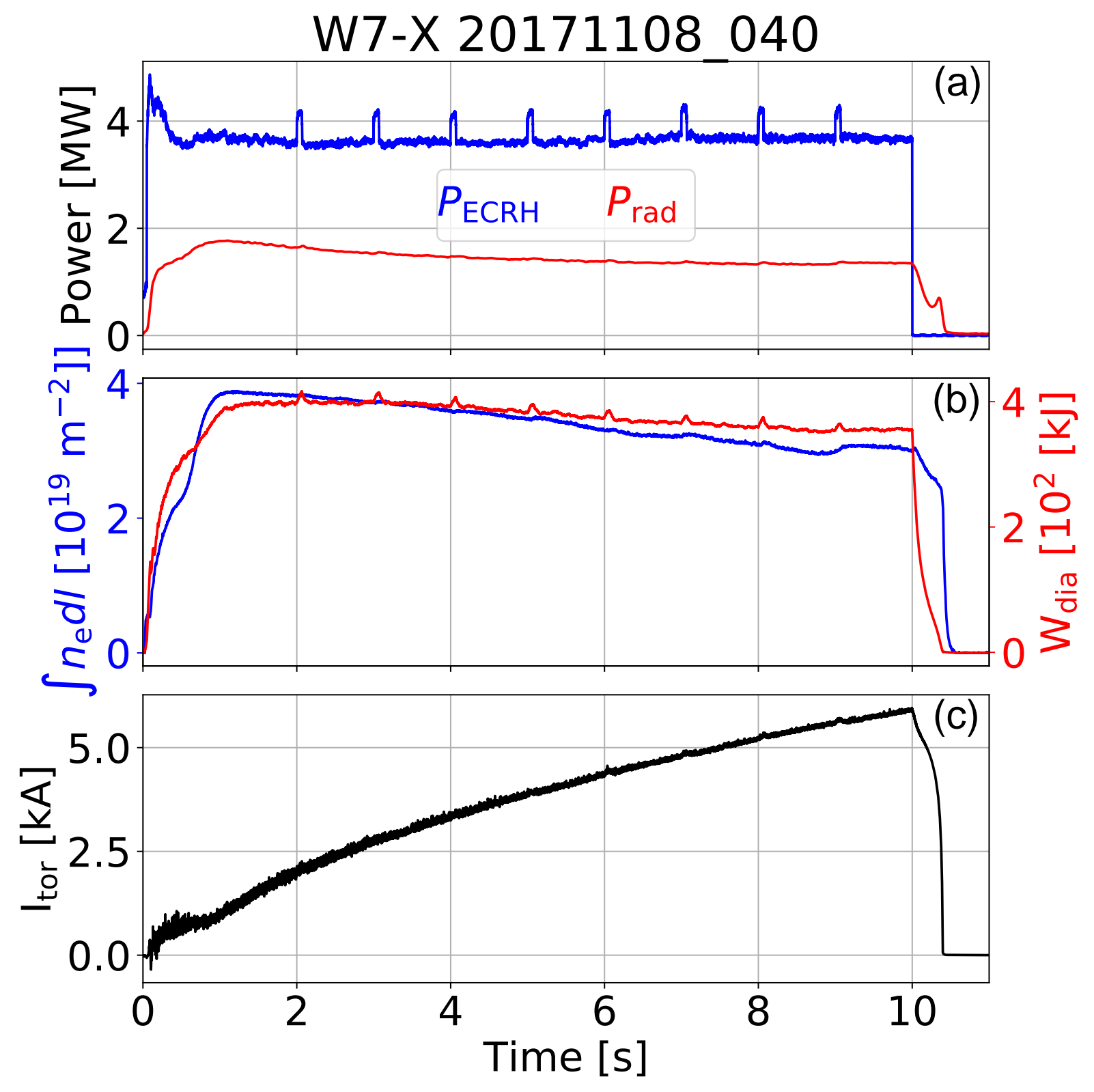

Figure 2: Overview of discharge 20171108_040. (a) The ECRH heating and radiation power. (b) The line integrated electron density measured by interferometer and diamagnetic energy by diamagnetic loop. (c) $I_{\text {tor }}$ measured by in-vessel Rogowski coil. 


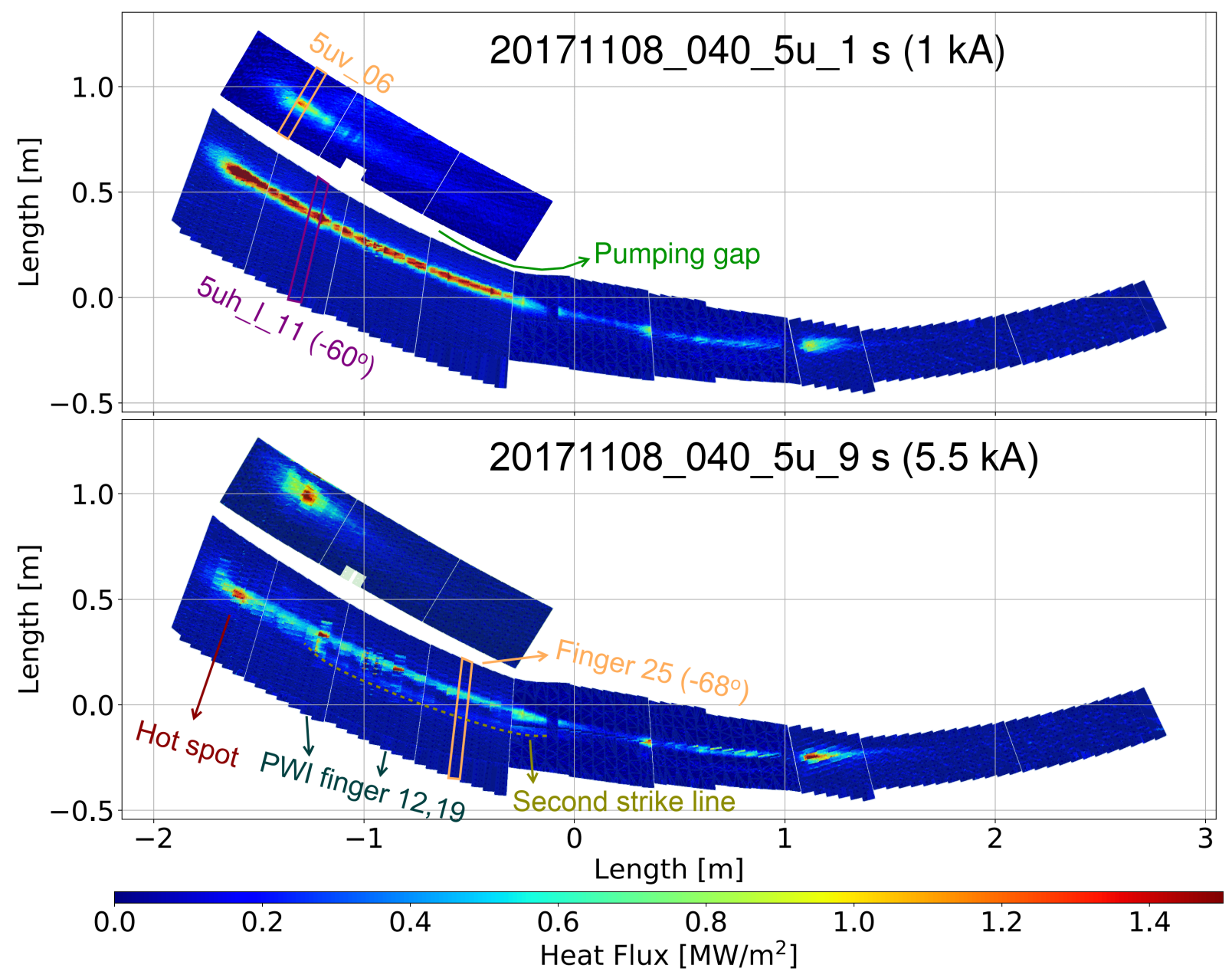

Figure 3: The heat flux distributions on the $5 \mathrm{u}$ divertor targets for discharge 20171108_040 at $1 \mathrm{kA}$ (top) and at $5.5 \mathrm{kA}$ (bottom). The indices of specific divertor elements (fingers) are marked for later analysis. The outer edge of the appeared second strike line is indicated by dashed line in the bottom sub-figure. 


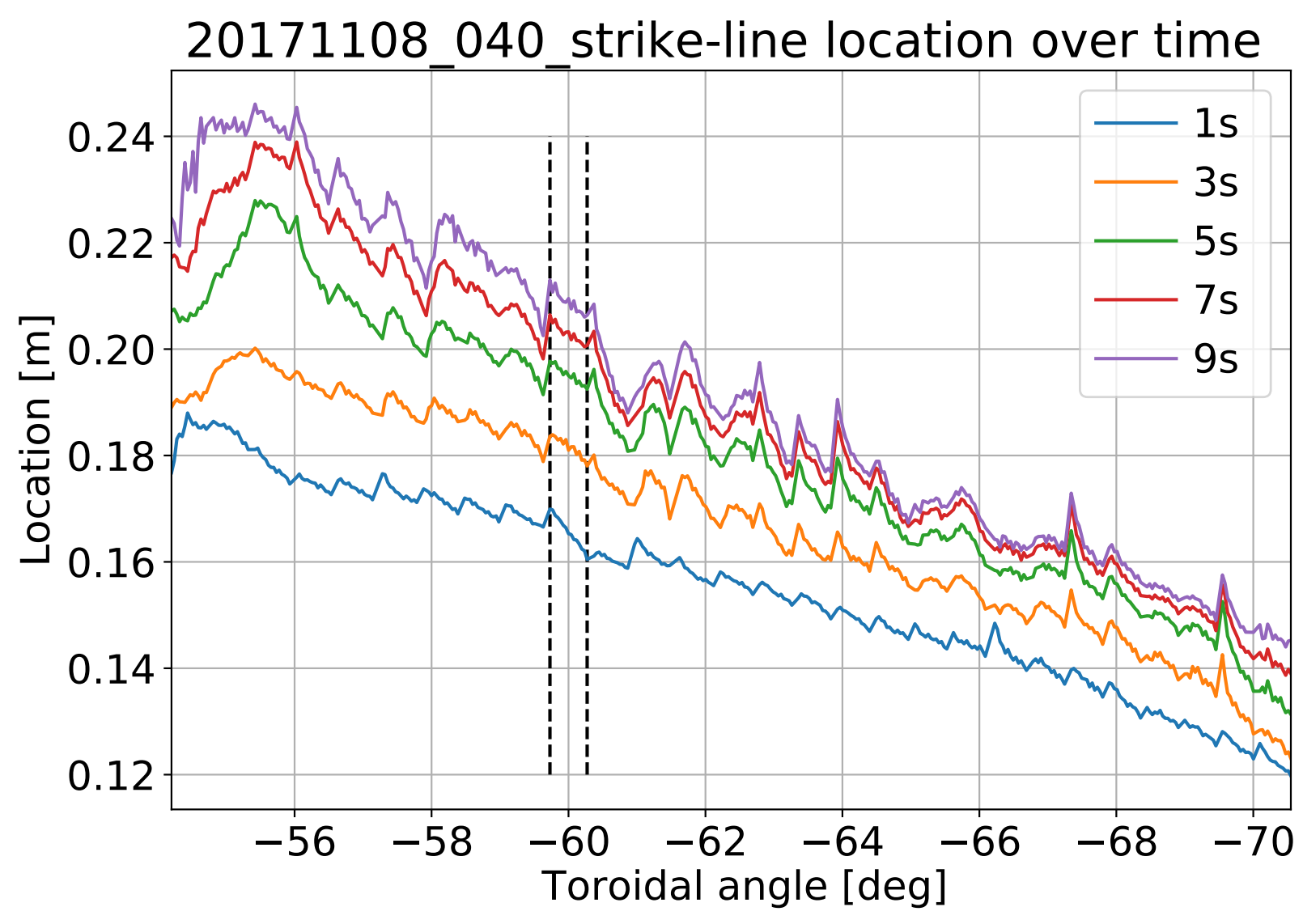

Figure 4: Temporal evolution of the strike-line location on the low-iota part of 5uh target. The $\mathrm{y}$ ordinate is the target coordinate of the local finger, while the $\mathrm{x}$ abscissa is the toroidal angle of the local strike line with $\Phi=-54^{\circ}$ from the lower iota end. The toroidal range for finger 5uh_l_11 is marked with dashed vertical lines.

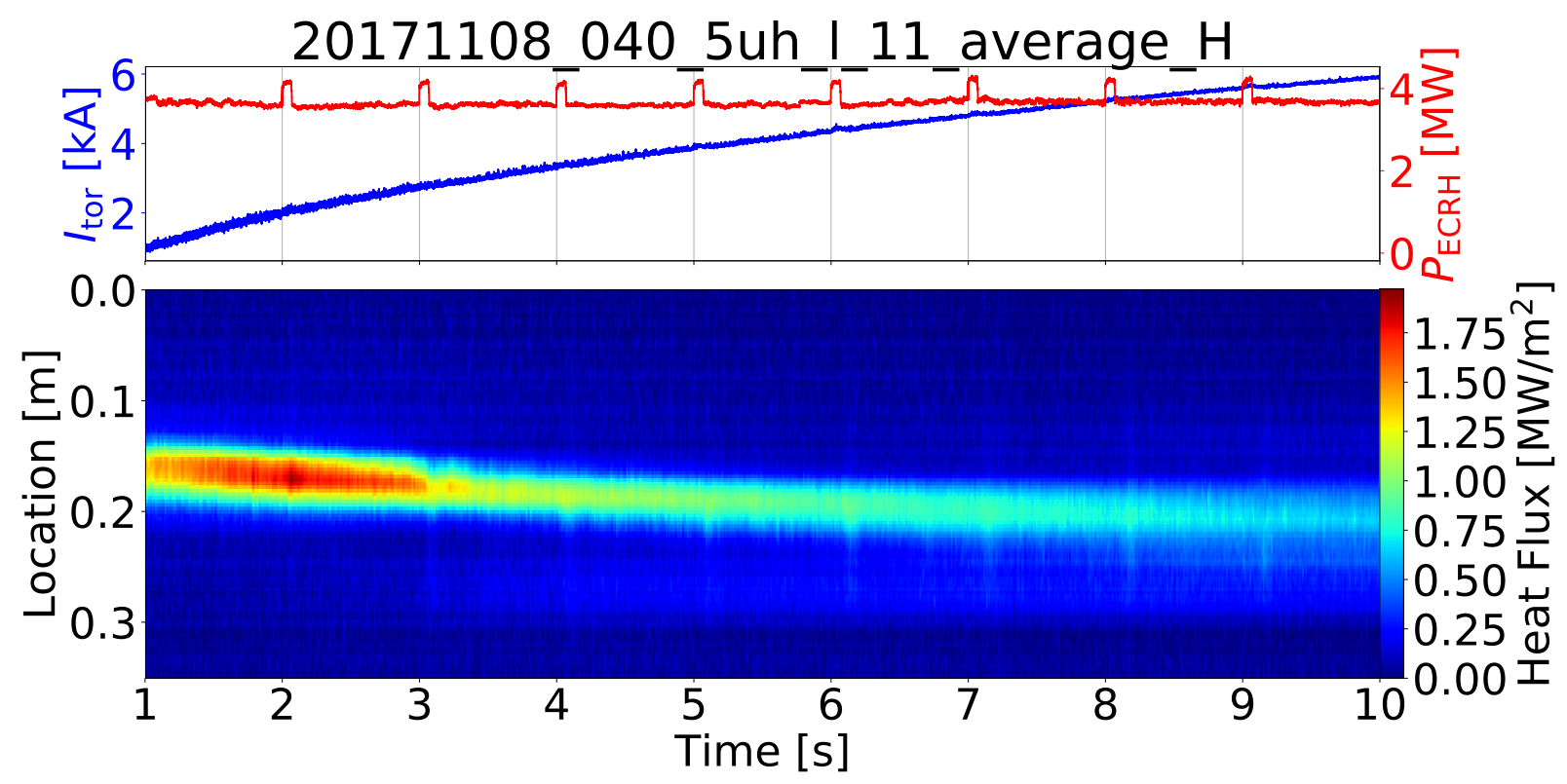

Figure 5: The time evolution of the averaged heat flux of finger 5uh_l_11 (bottom), ECRH heating power and $I_{\text {tor }}$ (top). 


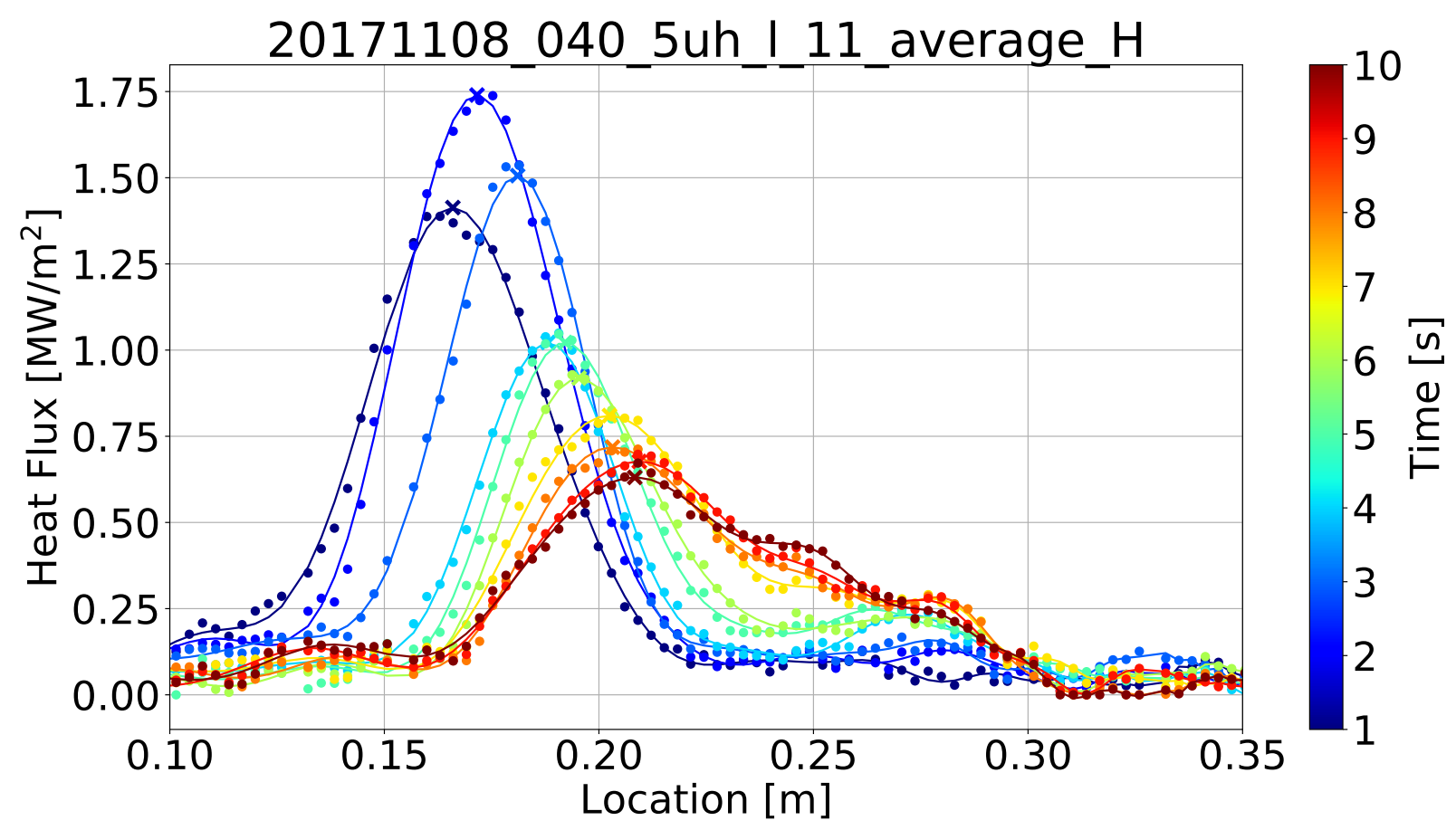

Figure 6: Ten averaged heat flux profiles of finger 5uh_l_11 from 1 to $10 \mathrm{~s}$. The experimental results are in dots, which are fitted with polynomial least square curve to locate the peak position (marked by a cross). The colorbar indicate the moments of the profiles. 


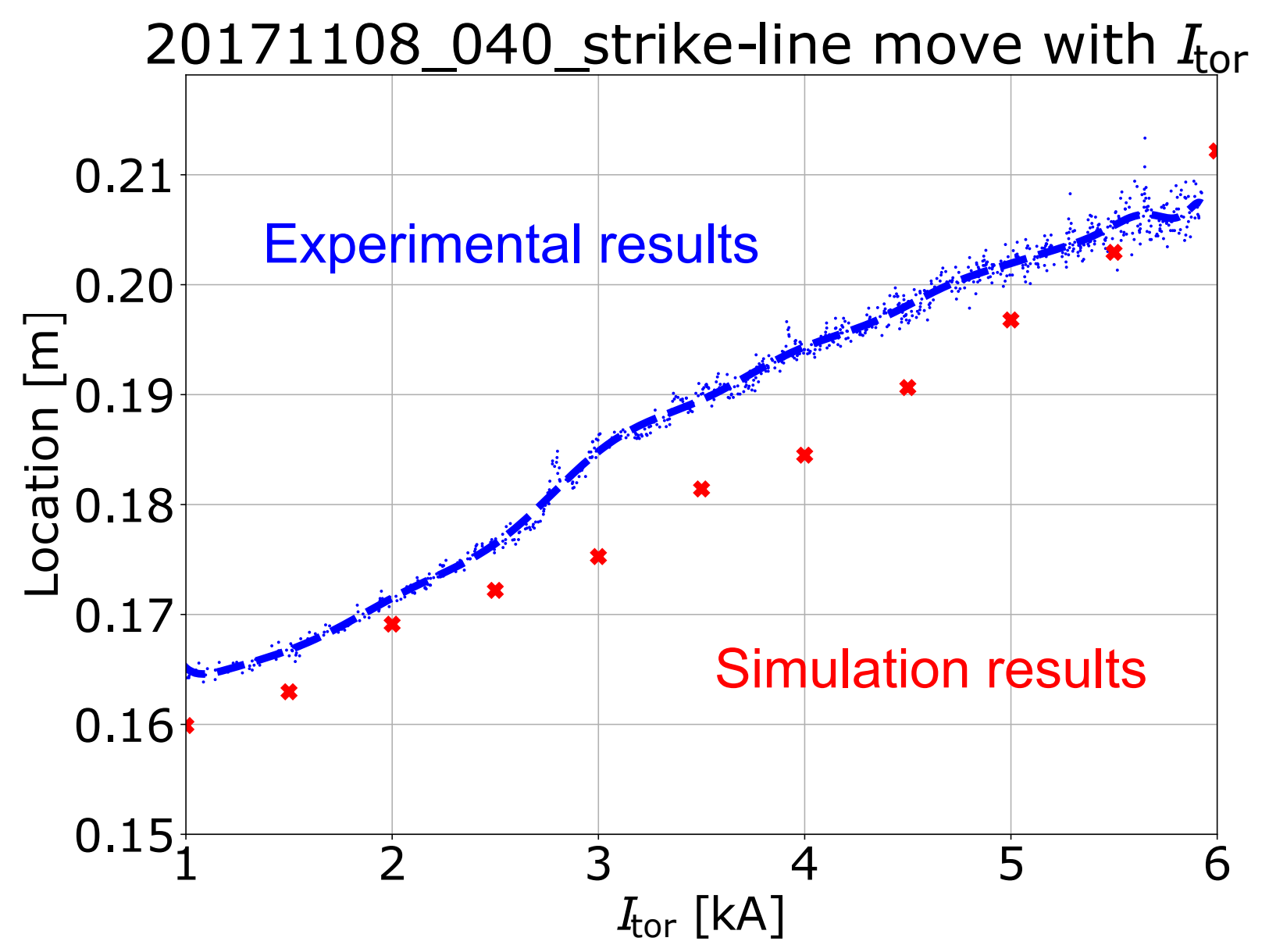

Figure 7: The comparison between the experimental results (blue) and simulations (red cross) for the strike-line movement against $I_{\text {tor }}$. The small blue dots are actual experimental data, which are fitted as dashed line to show the trend.

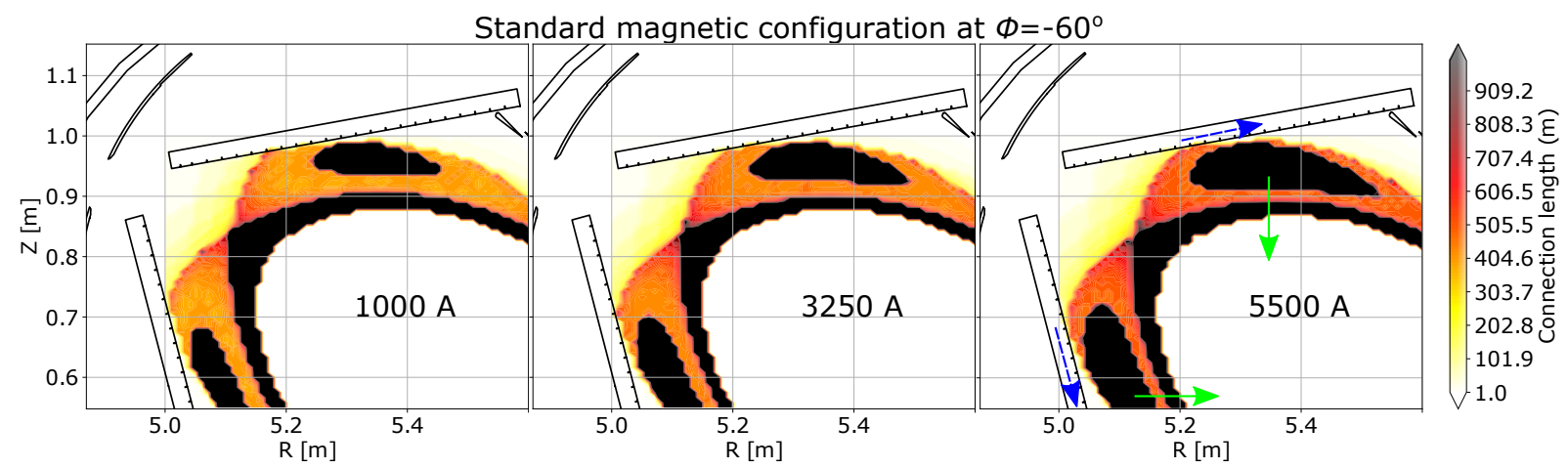

Figure 8: The $L_{c}$ plots with $I_{\text {tor }}$ of $1,3.25,5.5 \mathrm{kA}$ at toroidal angle of $\phi=-60^{\circ}$ for standard configuration. The green solid arrows indicate the shift of magnetic islands towards magnetic axis with increasing $I_{\text {tor }}$, while the blue dashed arrows show the resulting movement of the strike-lines. 


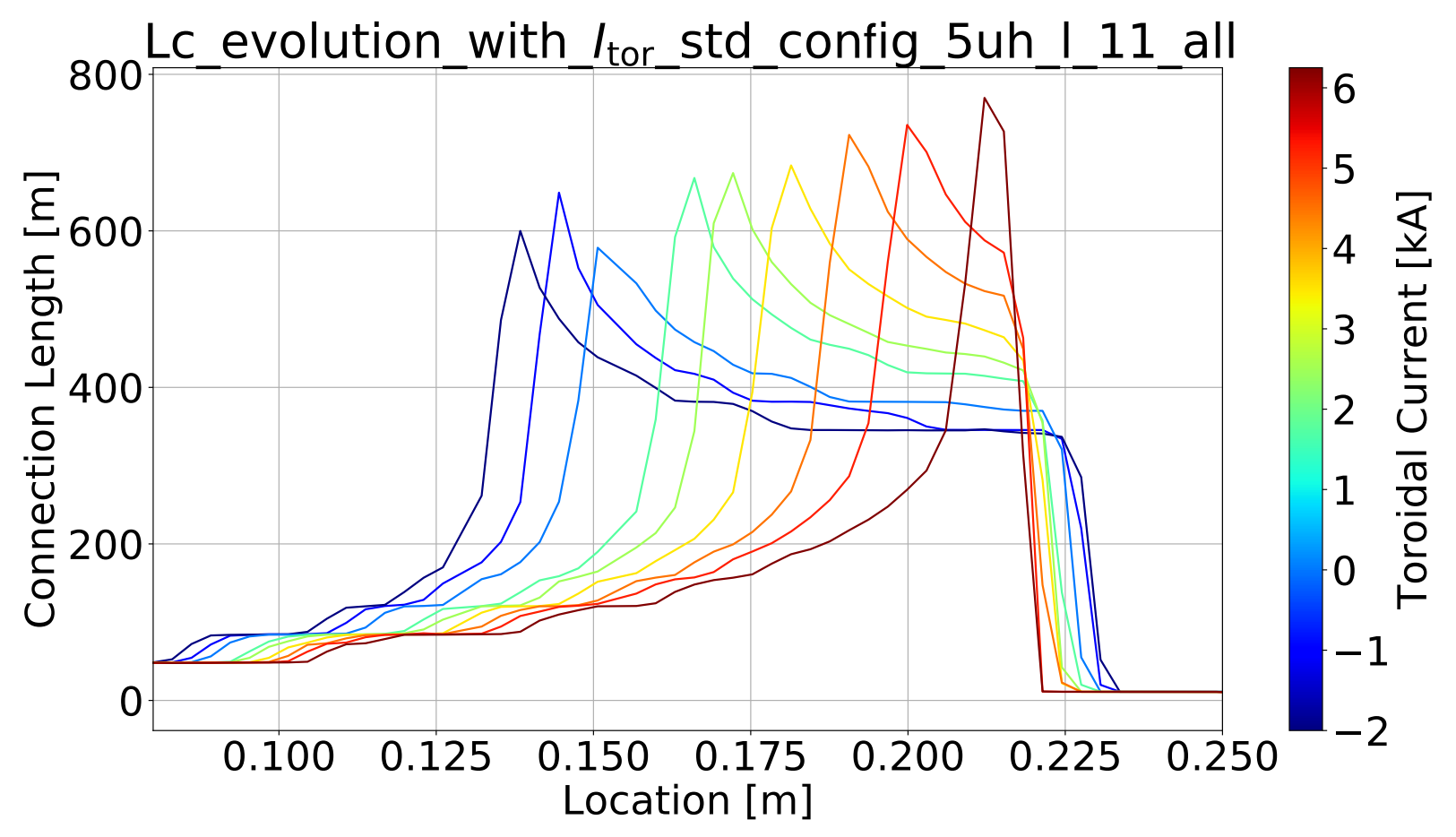

Figure 9: The $L_{c}$ profiles at finger $5 \mathrm{uh} \_$l_ 11 with different $I_{\text {tor }}$ from $-2 \mathrm{kA}$ to $6 \mathrm{kA}$ indicated by colorbar. 

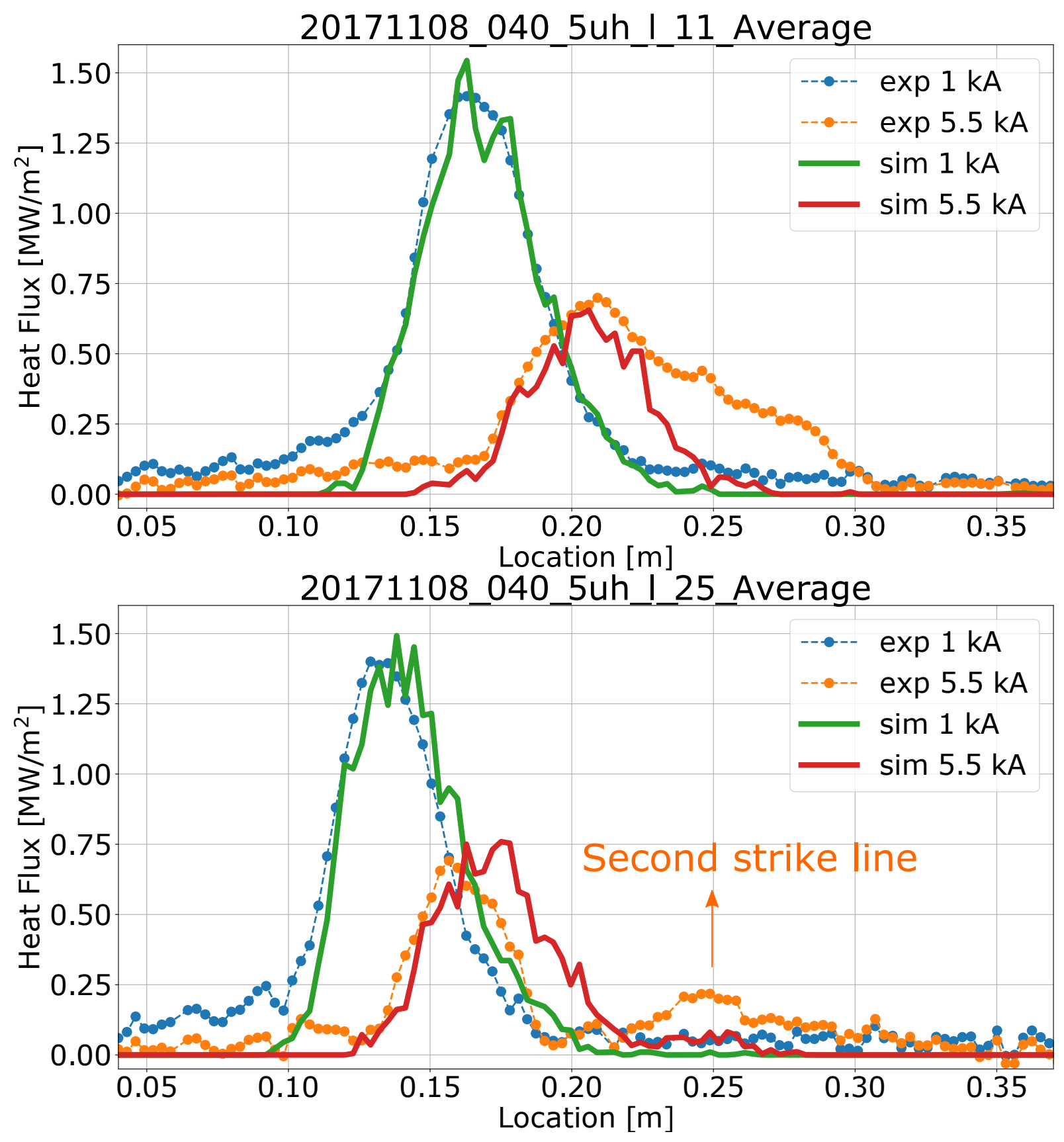

Figure 10: Comparison of experimental and simulated heat flux profiles with $I_{\text {tor }}$ of $1 \mathrm{kA}$ and $5.5 \mathrm{kA}$ for fingers 5uh_l_11 (top) and 5uh_l_25 (bottom). The positions of the fingers in the divertor are marked in figure 3. 


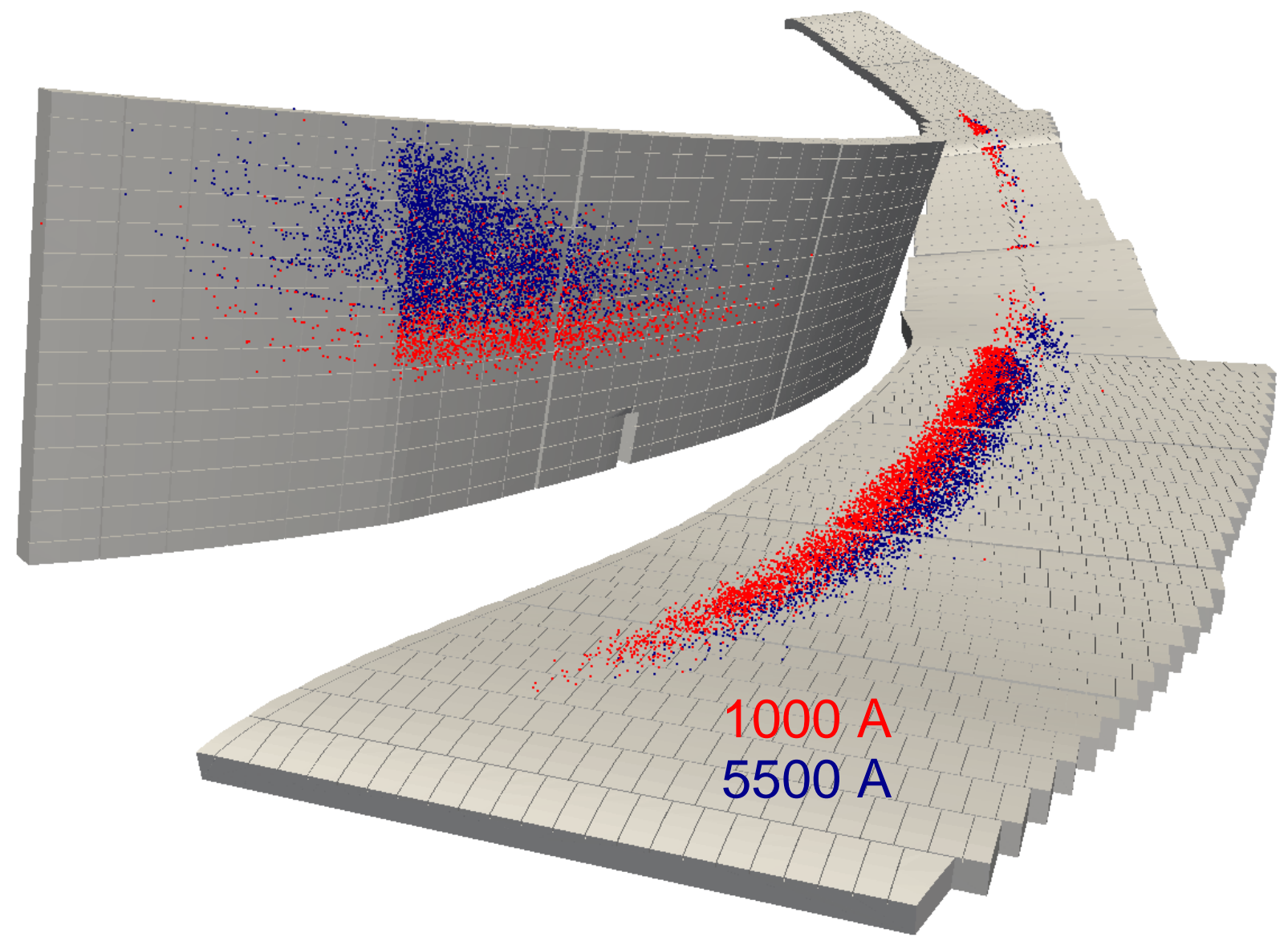

Figure 11: The resulting test particles on the divertor plates with diffusive FLTs including $I_{\text {tor }}$ of 1 (red) and $5.5 \mathrm{kA}$ (blue). 

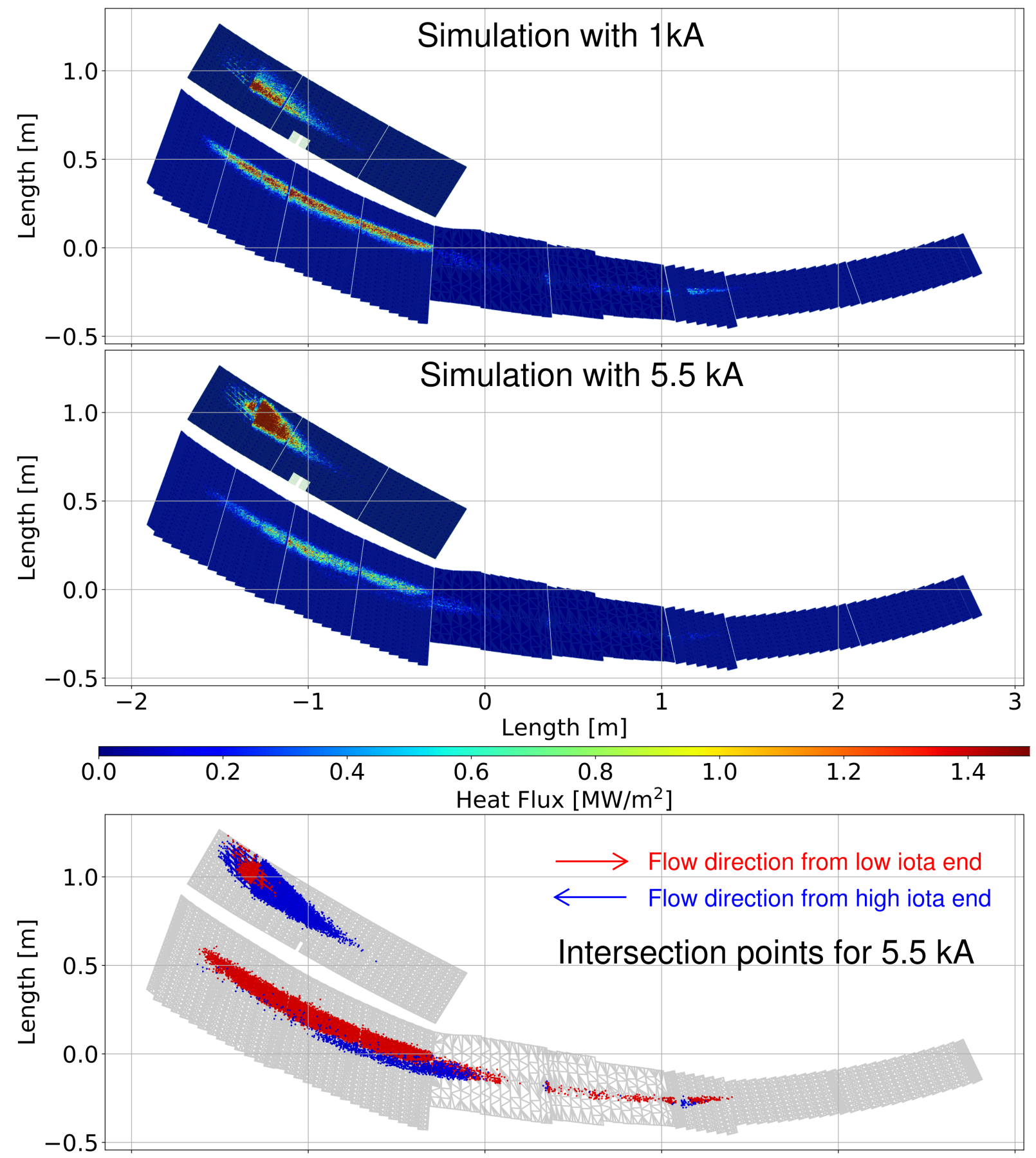

Figure 12: The simulated heat flux on the divertor plate with $I_{\text {tor }}$ of $1 \mathrm{kA}$ (top) and $5.5 \mathrm{kA}$ (middle), as well as the intersection points plot for $5.5 \mathrm{kA}$ case. 


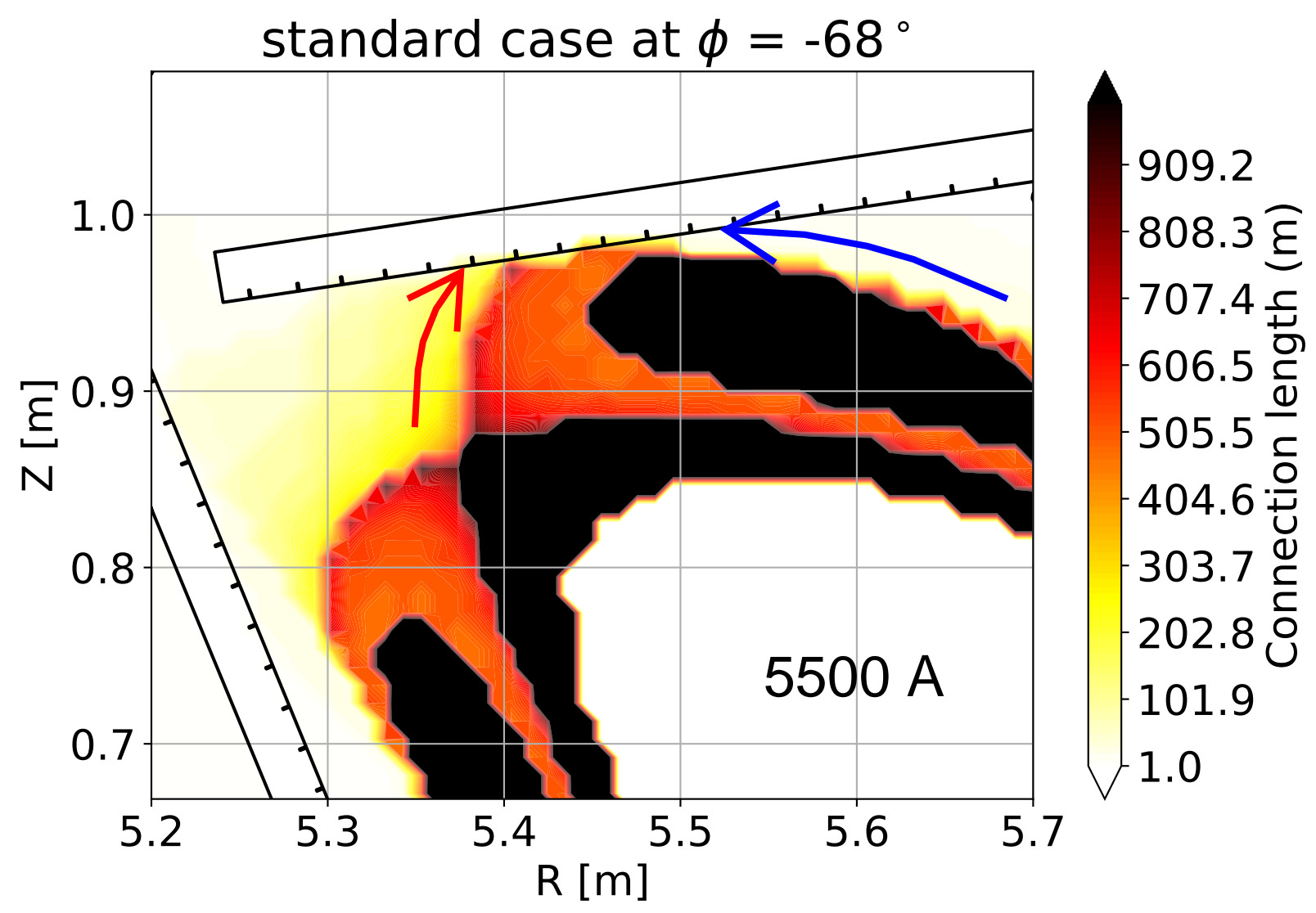

Figure 13: The $L_{c}$ plots with $I_{\text {tor }}=5.5 \mathrm{kA}$ at toroidal angle of $\phi=-68^{\circ}$ for standard configuration. The red arrow indicates the heat transport for the main strike line, while the blue shows the second strike line.

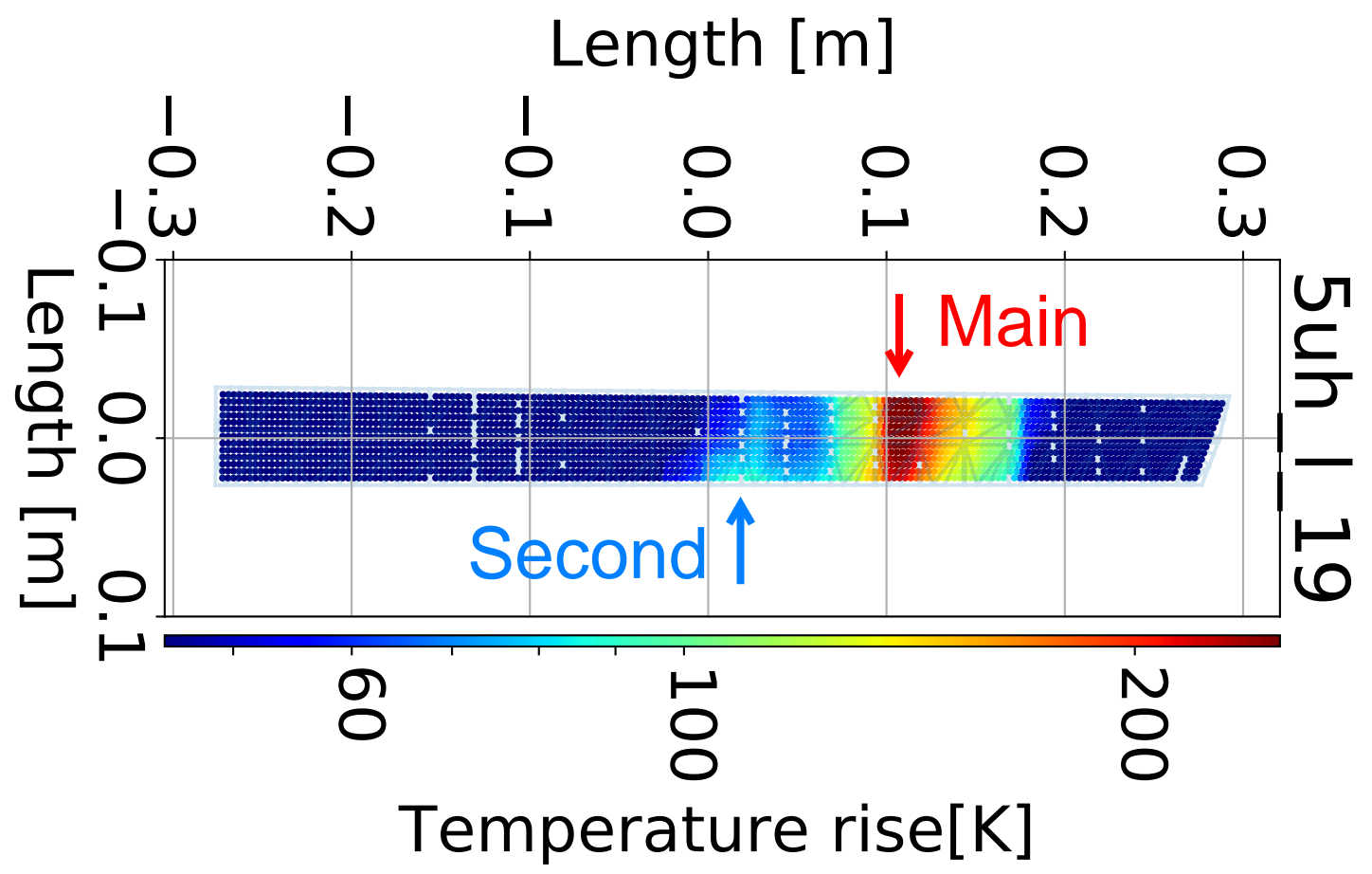

Figure 14: Distribution of the temperature rise on divertor finger 5uh_l_19 at $9 \mathrm{~s}\left(I_{\text {tor }}=\right.$ $5.5 \mathrm{kA})$. The main strike line and the second one are marked with red and blue arrows and labels, respectively. 


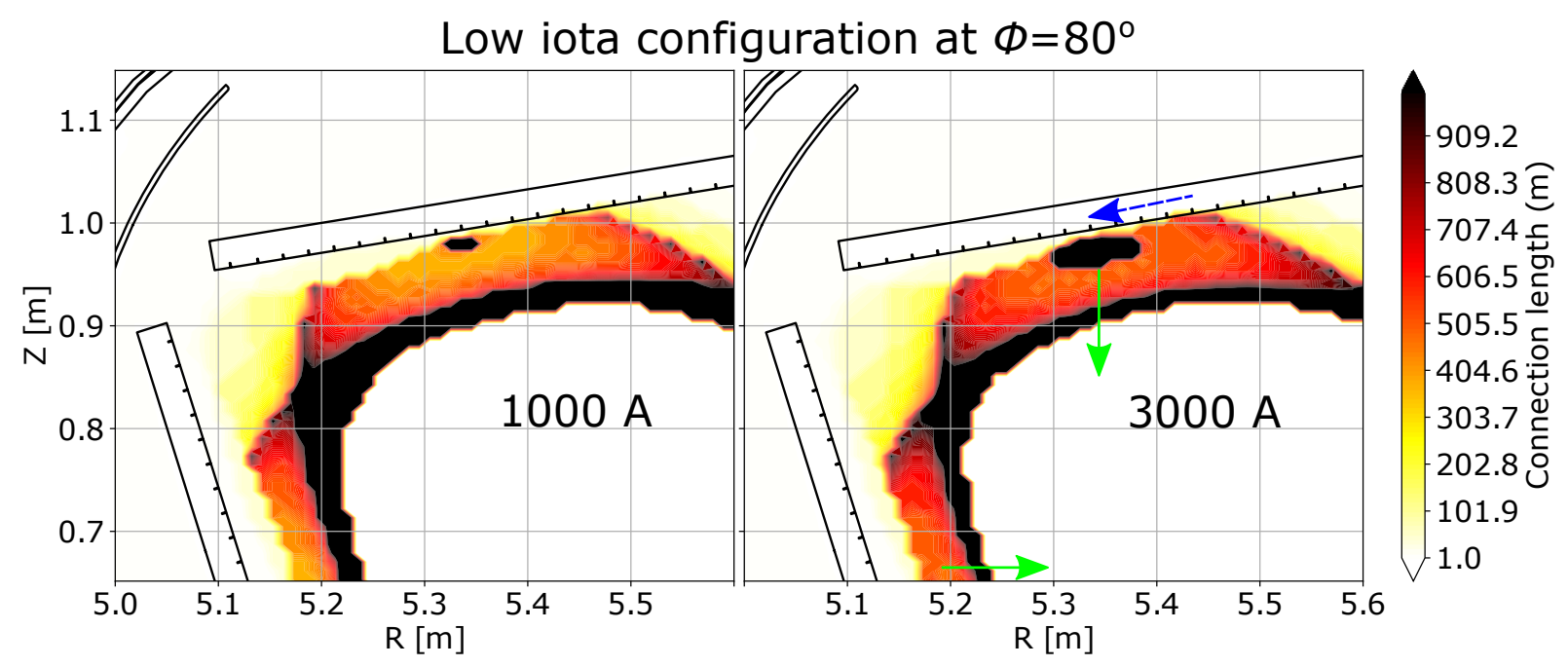

Figure 15: The $L_{c}$ plots with $I_{\text {tor }}$ of $1 \mathrm{kA}$ and $3 \mathrm{kA}$ at toroidal angle of $\phi=80^{\circ}$ for low-iota configuration.

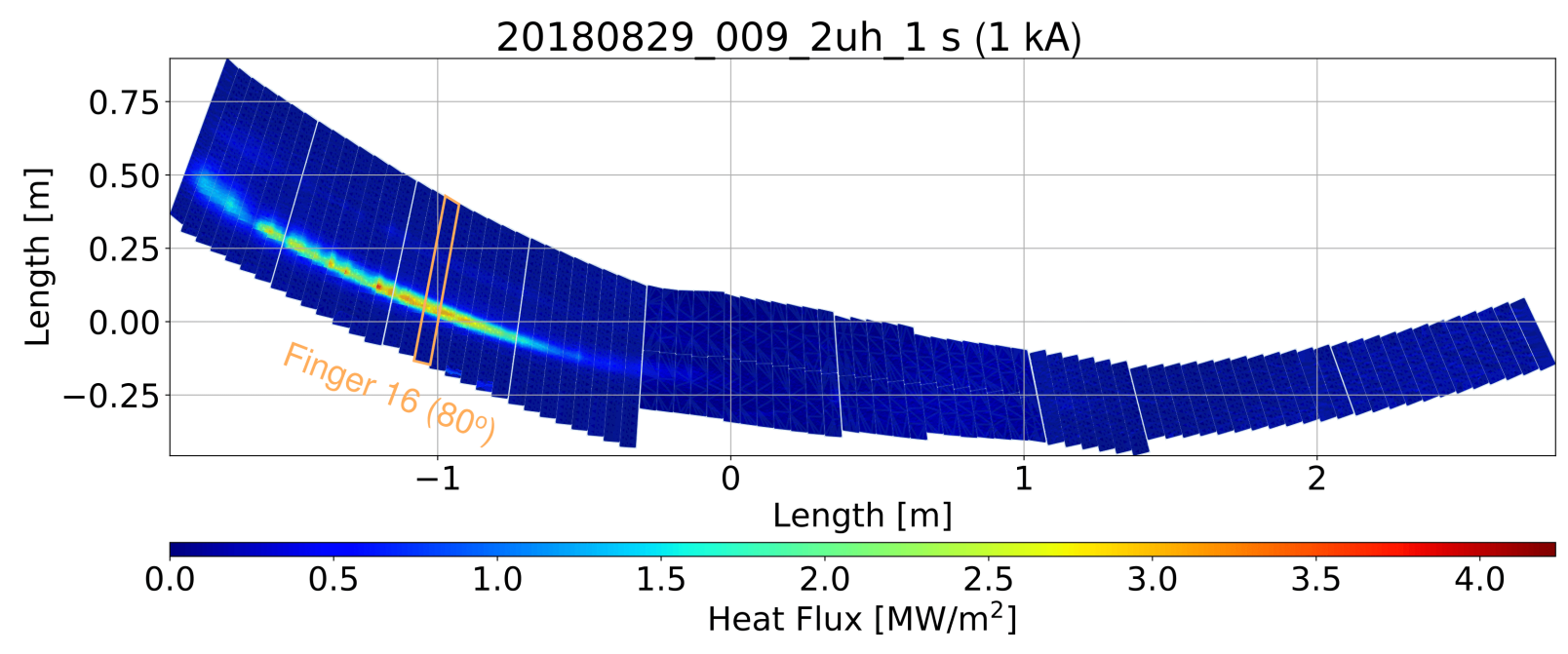

Figure 16: The divertor heat flux for discharge 20180829_009 on module 2 upper horizontal target at $1 \mathrm{~s}(1 \mathrm{kA})$. Finger 2uh_l_16 at toroidal angle $\phi=80^{\circ}$ is marked for other analyses. 


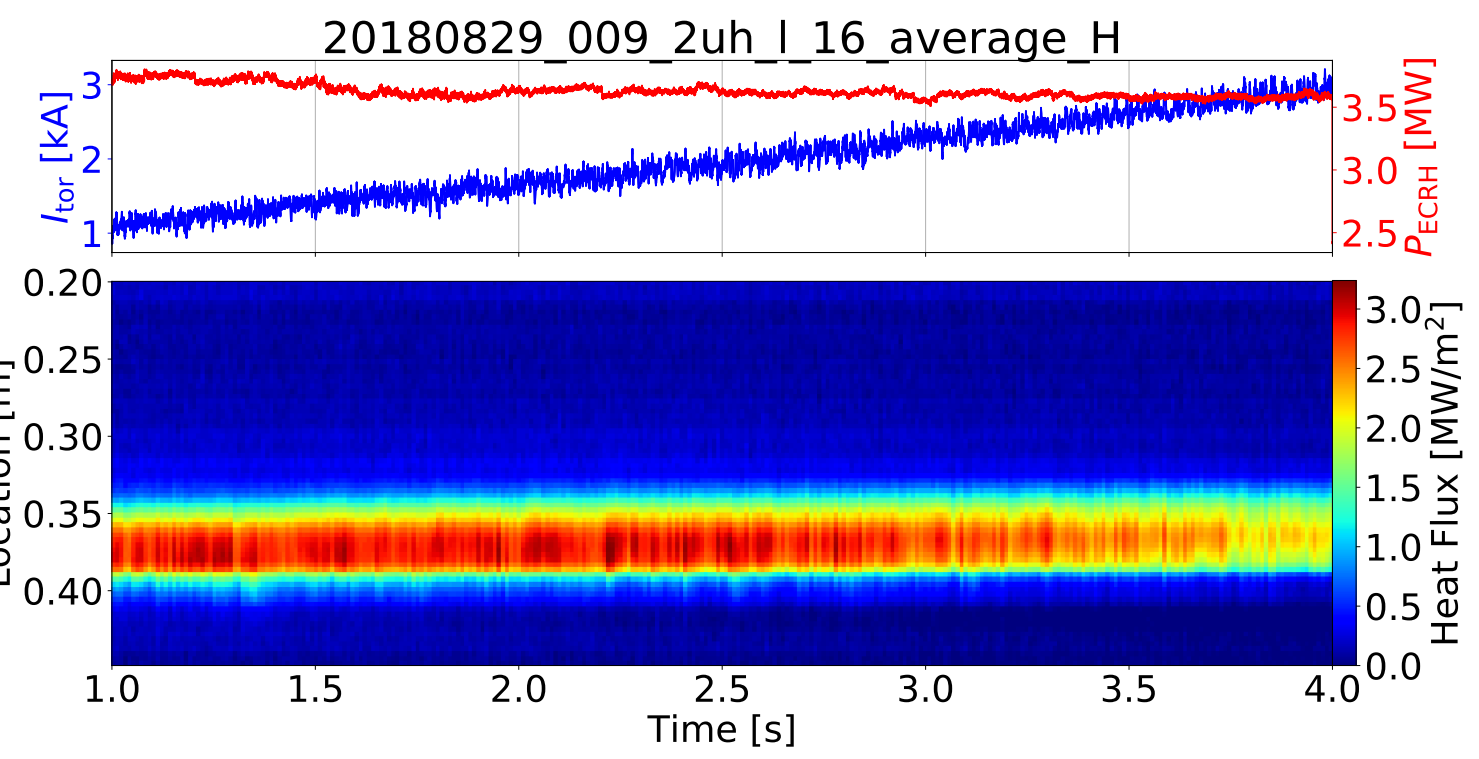

Figure 17: The time evolution of averaged heat flux of finger 2uh_l_16.

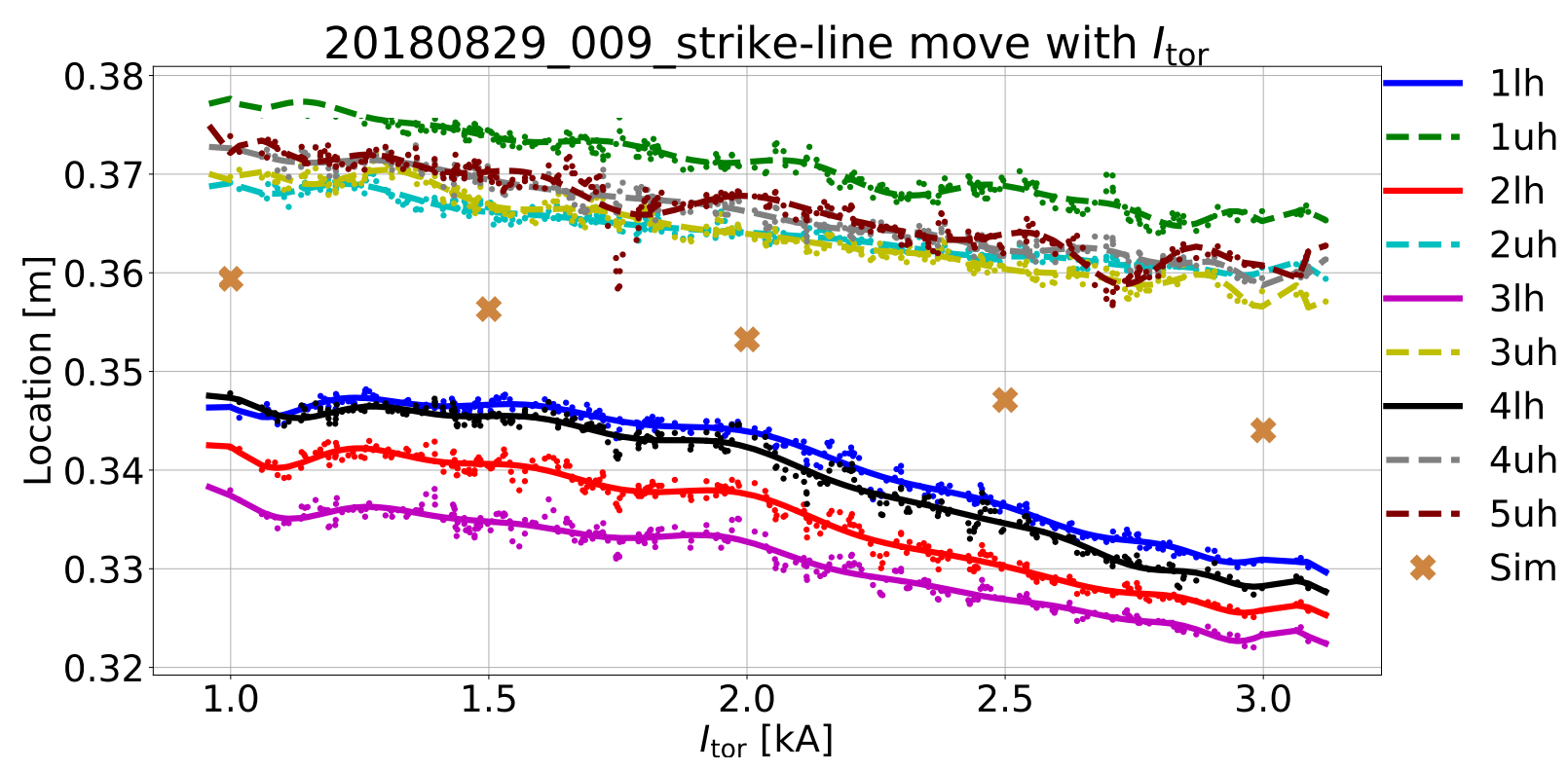

Figure 18: The strike line movements against $I_{\text {tor }}$ analyzed from nine IR cameras, and the comparisons with simulation (cross). Upper targets are marked with dashed line, while lower targets solid. 

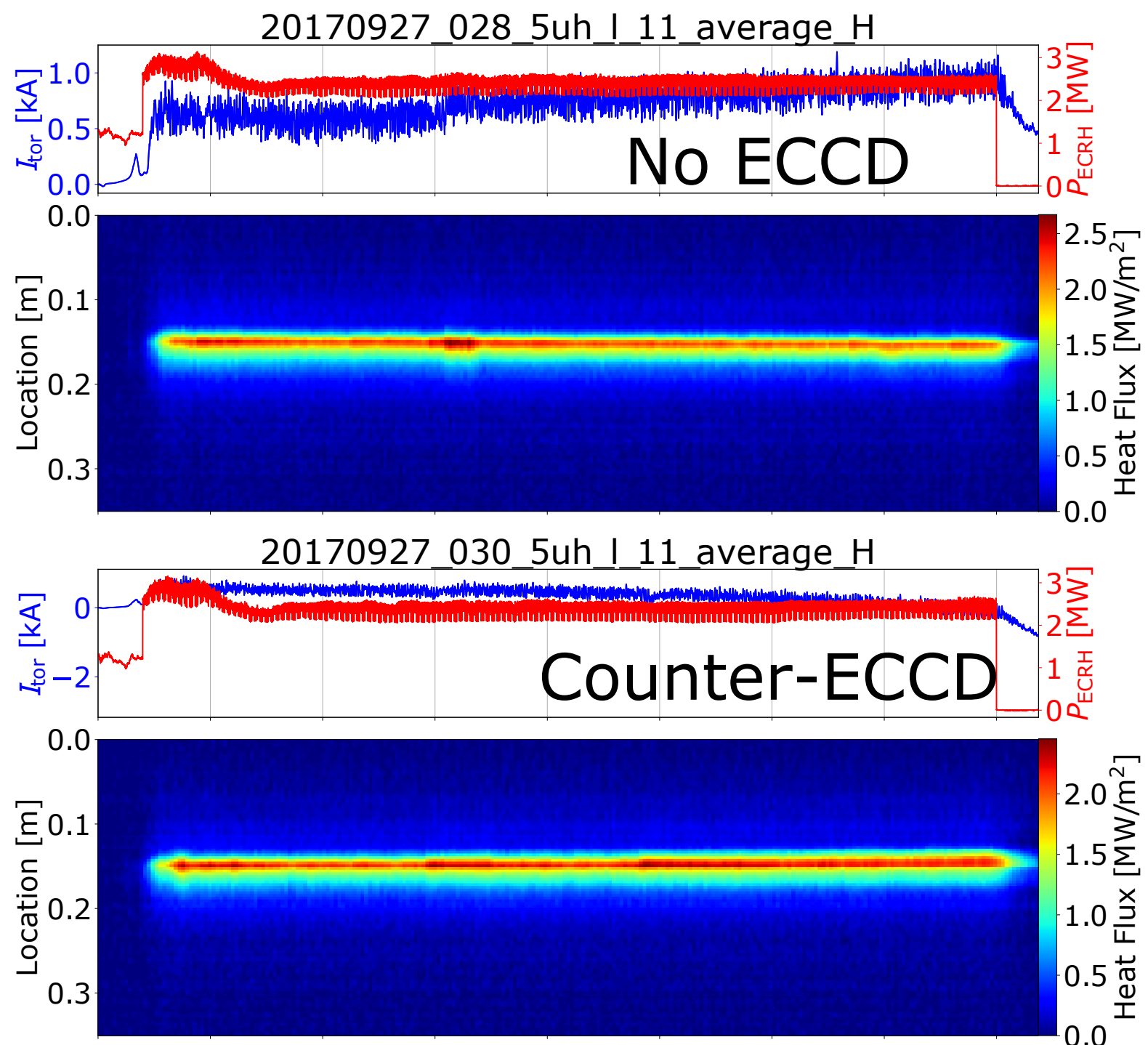

\section{7_042_5uh_I_11_average_H}
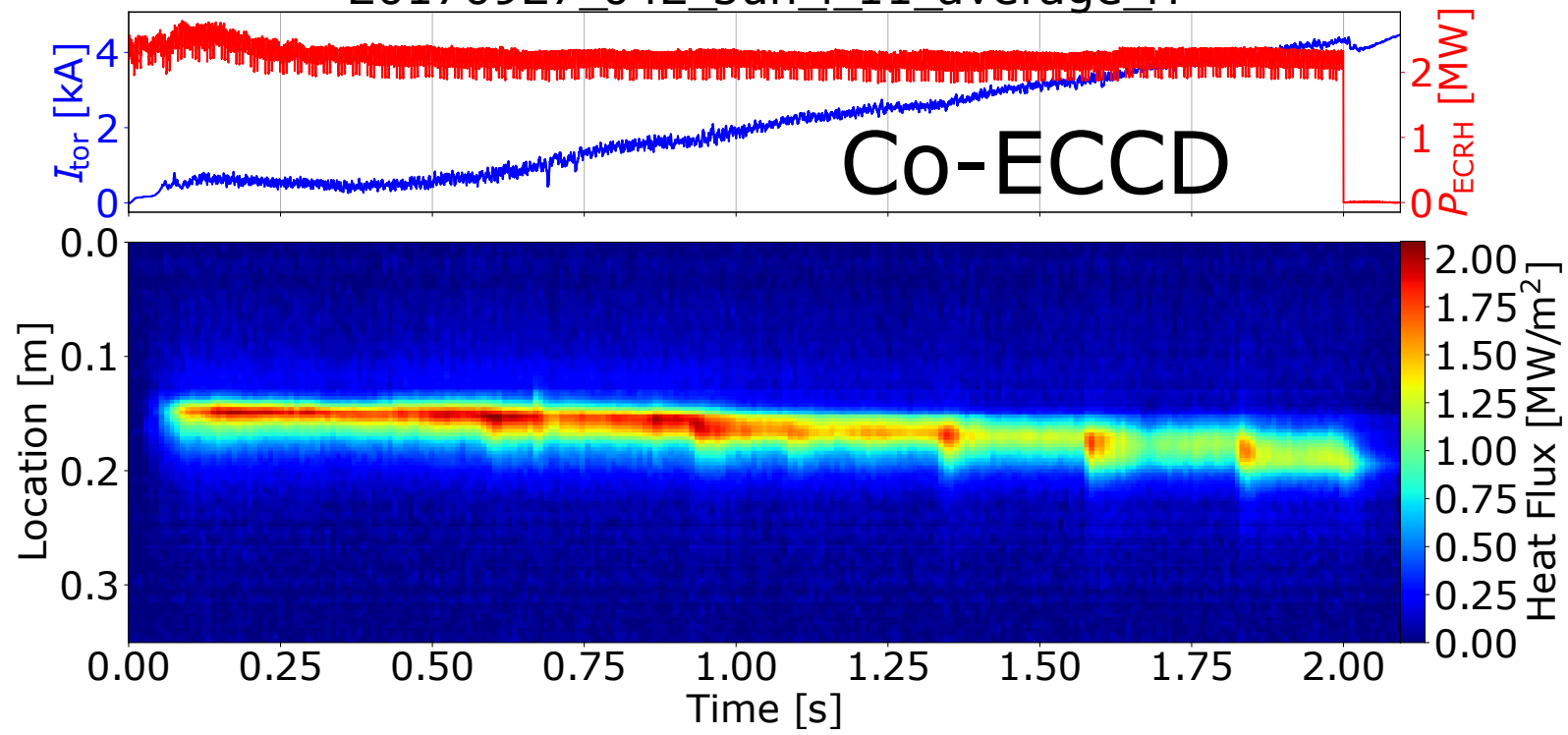

Figure 19: The time evolution of averaged heat flux of finger 5uh_l_11 for no ECCD discharge 20170927_028 (top), counter-ECCD discharge 20170927_030 (middle) and coECCD discharge 20170927_042 (bottom). 


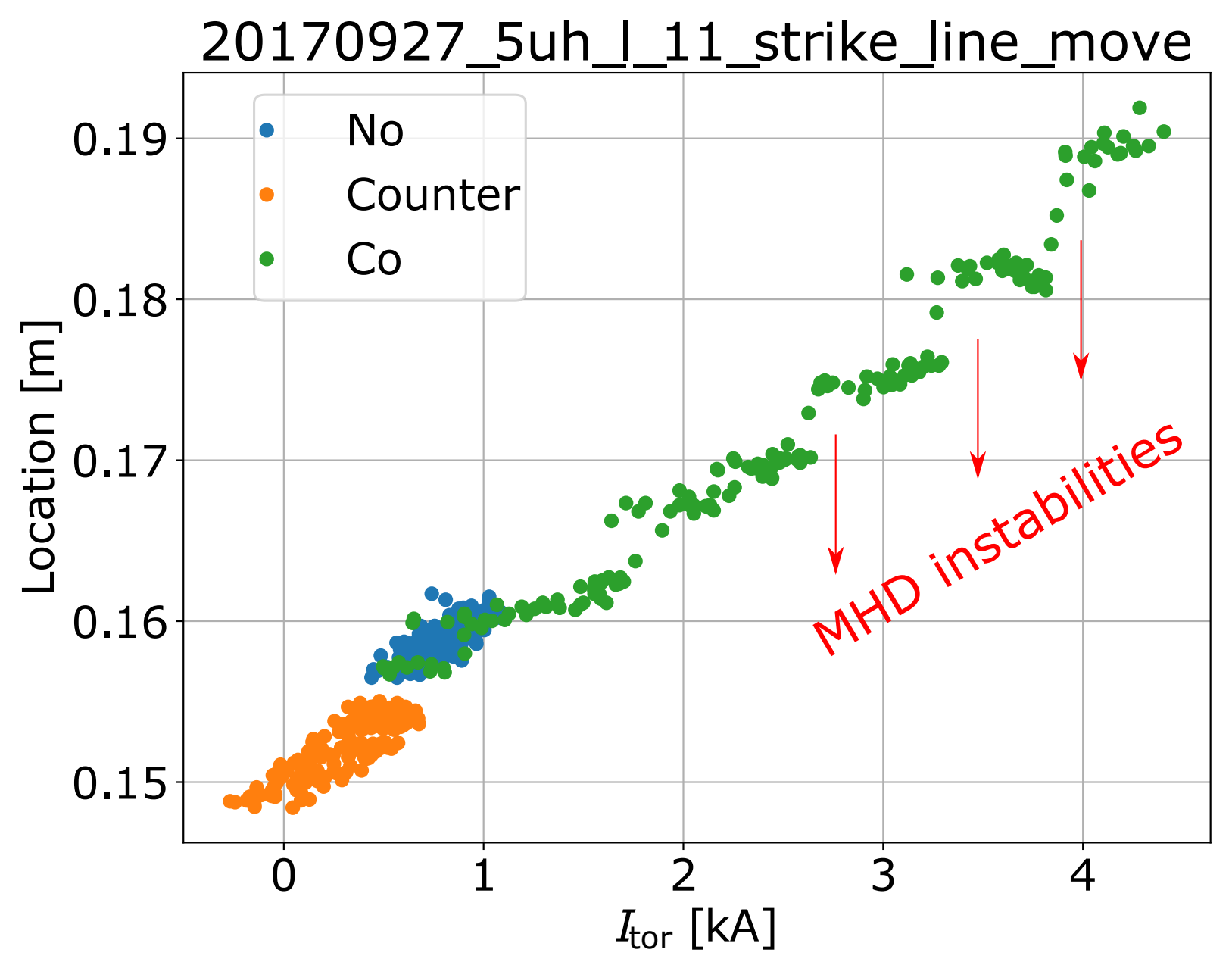

Figure 20: The strike line movement with $I_{\text {tor }}$ under no ECCD (blue), counter-ECCD (orange) and co-ECCD (green) cases. 